\title{
LPWAN Coverage Assessment Planning without Explicit Knowledge of Base Station Locations
}

\author{
Martin Stusek ${ }^{1,2}$, Dmitri Moltchanov ${ }^{2}$, Pavel Masek ${ }^{1}$, Konstantin Mikhaylov ${ }^{1,3}$, Jiri Hosek ${ }^{1}$, Sergey Andreev², \\ Yevgeni Koucheryavy ${ }^{2}$, Pavel Kustarev ${ }^{4}$, Otto Zeman ${ }^{5}$, and Martin Roubicek ${ }^{5}$ \\ ${ }^{1}$ Brno University of Technology, Faculty of Electrical Engineering and Communications, Dept. of Telecommunications, \\ Technicka 12, 61600 Brno, Czech Republic \\ ${ }^{2}$ Tampere University, Unit of Electrical Engineering, Korkeakoulunkatu 7, 33720 Tampere, Finland \\ ${ }^{3}$ University of Oulu, Center for Wireless Communications, Pentti Kaiteran katu 1, 90570 Oulu, Finland \\ ${ }^{4}$ ITMO University, Faculty of Software Engineering and Computer Systems, Kronverkskiy Prospekt, 49, St.Petersburg, Russia \\ ${ }^{5}$ Vodafone Czech Republic a.s., Namesti Junkovych 2808/2, 15500 Prague, Czech Republic \\ Contact author's e-mail: masekpavel@vutbr.cz
}

\begin{abstract}
An assessment of radio network coverage, usually in the form of a measurement campaign, is essential for multibase-station (multi-BS) network deployment and maintenance. It can be conducted by a network operator or its served consumers. However, the number of measurement points and their locations may not be known in advance for an efficient and accurate evaluation. The main goal of this study is to propose a new methodology for understanding the selection of measurement points during coverage and signal quality assessment. It is particularly tailored to multi-BS low-power widearea network (LPWAN) deployments without explicit knowledge of BS locations. To this aim, we first conduct a large-scale measurement campaign for three popular LPWAN technologies, namely, NB-IoT, Sigfox, and LoRaWAN. Utilizing this baseline data, we develop a procedure for identifying the minimum set of measurement points for the coverage assessment with a given accuracy as well as study which interpolation algorithms produce the lowest approximation error. Our results demonstrate that a random choice of measurement points is on par with their deterministic selection. Out of the candidate interpolation algorithms, Kriging method offers attractive performance in terms of the absolute error for NB-IoT deployments. By contrast, for Sigfox and LoRaWAN infrastructures, less complex techniques, such as Natural-neighbor, Linear interpolation, or Inverse-Distance Weighting, can achieve comparable (and occasionally even better) accuracy levels.
\end{abstract}

\section{INTRODUCTION}

\section{A. Background and Rationale}

The recent progress in miniaturization and automation has created a large application domain known as the Internet of Things (IoT) that has to be supported by a specific connectivity feature, named massive machine-type communication (mMTC). In response to these needs, a variety of low-power wide-area network (LPWAN) technologies, including LoRaWAN, Sigfox, and the third generation partnership project (3GPP)-ratified Narrowband IoT (NB-IoT), have been proposed. From the perspective of licensing, Sigfox represents a proprietary solution, while NB-IoT and LoRaWAN are open standards supported by the 3GPP initiative and LoRaWAN Alliance, respectively [1]-[3]. These systems are characterized by multi-kilometer communication ranges achieved over a relatively sparse deployment of base stations (BS)s. As the range of applications includes smart home scenarios as well as industry-driven services for remote metering and sensing, radio connectivity has to be enabled in outdoor as well as deep indoor environments [4], [5].

Nonetheless, before deploying LPWAN communication technologies (which should be valid for any wireless system), radio network planning is employed to produce an initial estimate on coverage and signal quality. However, this is a complex process affected by various factors and constraints including non-technological aspects, such as types of urban layout, legal issues, and selection of locations where the BS can be deployed. In practice, a decision on the BS placement is made based on the propagation modeling that uses threedimensional city maps. Such a process may involve multiple iterations, where radio measurements are used to adjust the locations of the installation points. Even after the deployment, network operators conduct regular inspections to understand whether changes in the propagation environment have affected the coverage characteristics and the performance of their networks [6]-[8].

Our previous works [9], [10] also confirm the presence of dynamic fluctuations over extended periods of time. Two measurement campaigns conducted during several monthslong intervals revealed that the fluctuations can be as high as $40 \mathrm{~dB}$ in terms of the signal strength even for static deployments. These changes are not entirely random, but they are represented by a slow variation of samples oscillating around the mean value. On the other hand, micro-scale variations in the signal strength such as fast fading are smoothed using the "averaged" propagation models.

As a result, to maintain a relevant coverage map, the said process has to be applied periodically. The rationale is that LPWAN network operators may perform upgrades of their infrastructures or reconfigurations of the network parameters while the environmental conditions may also change with time due to, e.g., new construction in the considered area or seasonal changes. We also note that the duration of time when the propagation environment remains relatively unchanged depends on many factors and is, in general, site-specific; thus, 
beyond the scope of this work. Also, the coverage map update period is a valid research consideration on its own, which we leave for further studies.

On top of that, conducting a measurement campaign on the city scale is a time-consuming process. Preferably, the operators are willing to assess the network coverage and signal quality in all the available locations. One of the crucial issues in evaluating the network coverage is that the number of measurement points required for an accurate coverage and signal quality reconstruction is not straightforward to determine. In practice, it is affected by the city deployment features, selected technology, carrier frequency, etc. This problem becomes even more complex when the exact locations of the BSs are not known in advance. The latter might be the case for third-party companies aiming to purchase a service from the LPWAN operators and performing their own coverage and signal quality assessments.

The recently proposed fully-automated coverage assessment techniques, such as those utilizing unmanned aerial vehicles (UAVs) [11]-[15], may be constrained either due to the weight of the measurement equipment or with respect to allowable altitudes above the ground, and may thus produce inaccurate or limited results at the evaluated points. On top of this, in some regions, the use of UAVs for such measurements may be prohibited or hampered by the legal regulations.

\section{B. Key Contributions}

In this paper, we propose a new methodology for assessing the coverage and the signal quality of multi-BS LPWAN technologies. Specifically, based on the results of our extensive measurement campaign, we address the problem of signal quality estimation at the feasible points within a certain area of interest. We then quantify how the number of measurement locations affects the accuracy of estimation and how well different interpolation and thinning algorithms perform. Notably, in our study, we consider all three popular LPWAN technologies dominating the market today, namely, NB-IoT, Sigfox, and LoRaWAN. This allows us to understand how the technology features affect the coverage and the signal quality assessment procedure in city-wide LPWAN layouts.

In the first phase of this study, our joint effort with Vodafone Czech Republid 1 delivers (i) an extensive measurement campaign for three leading LPWAN technologies publicly available today (wherein the research activities with Vodafone allowed us to adjust the network configuration as well as obtain detailed information while performing the measurement campaign), across the city of Brno located in the Czech Republic [16]-[19]. Using these measurements and utilizing the knowledge of the BS locations for all the technologies in question, (ii) we construct the baseline coverage map and derive a reference model used further on to characterize the prediction accuracy of the propagation models as an alternative to the interpolation methods.

\footnotetext{
${ }^{1}$ Research cooperation between Vodafone Czech Republic and the Department of Telecommunications at Brno University of Technology has started in 2017, see: Deployment of the NB-IoT technology in Czech Republic.
}

In the second phase of our work, we consider exclusively the results of our practical measurements and proceed by gradually reducing the number of points taken into account randomly or deterministically, while at the same time applying appropriate interpolation techniques to (iii) create reduced coverage maps. Considering the proportion of the area covered with at least a certain signal level $(x \mathrm{dBm})$ and employing the weighted average metric, (iv) we compare the performance of the reference model against the coverage maps produced by the interpolation algorithms. Finally, (v) we assess the performance of the reference model and the interpolation methods under thinning against the measurement results.

With the proposed methodology, one may identify the number of measurement points required to produce a coverage assessment with the desired accuracy for a city environment similar to the one of Brno, and can also deduce useful insights for other environments. In a nutshell, the main contributions of our study are:

- we propose a methodology for identifying the number of measurement points and their locations to conduct a coverage assessment of LPWAN technologies at city scale with a given approximation error;

- we show that out of a large set of the considered interpolation techniques, Kriging provides the best performance for the NB-IoT deployments, while Naturalneighbor interpolation can become a viable alternative for the LoRaWAN and Sigfox deployments;

- we establish that the number of measurement points required to achieve a certain coverage and signal quality assessment error increases with the density of the BSs and also depends on the communication range of the considered radio technology;

- we demonstrate that a random choice of measurement points produces a similar approximation accuracy as their deterministic selection.

The rest of this paper is organized as follows. In Section we overview the related work. Further, in Section III, the measurement campaign and the obtained results are described. Our proposed methodology for identifying the minimum sets of measurement points for an accurate characterization of the radio coverage is introduced in Section IV The key numerical findings are summarized and discussed in Section V Finally, the conclusions are drawn in Section VI

\section{RELATED WORK}

Accurate network coverage assessment is one of the most challenging operations that precede a deployment of the end devices (ED)s, which rely on wireless communications technology [20]. Field measurements provide a method of coverage characterization, but conducting these is a costly and time-consuming process. Therefore, by limiting the required number of measurement points needed to obtain accurate coverage and signal quality results, major technology adopters can save a significant amount of resources in terms of time and money. 
Today, the assessment of radio coverage is even more important as many industrial companies were waiting for the 3GPP cellular IoT (CIoT) technologies to hit the market. Therefore, the key players are now preparing their first largescale LPWAN trials and challenge the operators to deliver a reliable network infrastructure capable of managing the massive numbers of connected devices. To the best of our knowledge, the research question of assessing large-scale deployments of multi-BS LPWAN technologies without the knowledge of BS locations has not been sufficiently investigated in the literature as of yet. In the following subsections, (i) we first summarize past studies related to coverage assessment; (ii) we then overview the state-of-the-art interpolation methods used for coverage estimation; and (iii) we finally summarize the standard coverage assessment metrics.

\section{A. Coverage and Signal Quality Assessment}

Coverage and signal quality assessment of LPWAN technologies with explicit knowledge of BS locations was addressed in several research works. Most of these studies, however, are limited to a single channel quality indicator, i.e., reference signal received power (RSRP), received signal strength indicator (RSSI), or signal-to-interference plus noise ratio (SINR), thus making a comparison between different works cumbersome. Below, we report on the recent measurement setups over city-scale deployments.

In [21], the authors carried out an extensive measurement campaign, wherein coverage assessment data acquired for LoRaWAN and Sigfox served as an input set for the proposed localization framework. The study was conducted in the city of Antwerp, Belgium. With 84 BSs distributed over the area of $52.97 \mathrm{~km}^{2}$, Sigfox slightly exceeds LoRaWAN in terms of the number of gateways $(\mathrm{GW}) \mathrm{s}$. For Sigfox, the authors also conducted a coverage assessment in rural environment between the cities of Antwerp and Ghent. This measurement campaign covered the total area of $1068 \mathrm{~km}^{2}$ featuring 137 BSs. Unfortunately, there is no publicly available NB-IoT measurement trial focusing on coverage and signal quality assessment at such a large scale.

In [22], researchers characterized the distribution of longterm evolution (LTE) BSs in the city of Xian, China based on real-world measurements. The results of the conducted measurement campaign can offer first-order insights into the density of NB-IoT BSs. In this urban scenario, 13 BSs cover the area of $3 \mathrm{~km}^{2}$. The density of LTE BSs in [22] is comparable with the measurement results of our work.

Some other research works employed computer simulations to estimate coverage in LPWAN systems. In [23], the authors used existing Telenor's cellular deployment structure to model the coverage performance of NB-IoT, LoRaWAN, and Sigfox. The structure of the network suggested that the density of urban cells is five times higher as compared to rural areas. Further, simulation results confirmed that the outages over NBIoT and Sigfox do not exceed $1 \%$, followed by LoRaWAN with a $2 \%$ chance of message loss.
It is essential to highlight the fact that all the aforementioned studies presume full knowledge of the BS locations. Hence, these results can be used by the operators to assess and improve their deployments. However, this approach is not suitable for third parties, which cannot directly access the BS location data.

\section{B. Interpolation Methods}

One of the crucial steps in assessing large-scale deployments is the use of prediction mechanisms to interpolate the signal quality at those points where no measurement data is readily available. Several studies followed this approach. Particularly, in [24], the authors utilized IDW interpolation to predict LTE signal quality at locations with no measurement data. However, the authors of that publication did not propose any assessment metric. Hence, the accuracy of the results cannot be verified, and the predicted value is considered as "ground truth".

The coverage assessment in [25] employed fixed rank Kriging (FRK) to predict the signal level in the region covered by a single macro-cell. The results indicated reasonable accuracy of that approach with the root-mean-square error (RMSE) ranging between 3 and $5 \mathrm{~dB}$. Further, the study in [22] proposed a new method of coverage map construction based on multicriteria triangulation-induced interpolation (MTI). However, that work focused only on the covered area prediction without closer specification of the expected signal levels.

Finally, in [26], the authors utilized Linear, Nearestneighbor, and IDW interpolation schemes together with Minimum, Mean, Gradient, IDW, and Nearest-neighbor extrapolation algorithms to construct their received signal strength (RSS)-based localization framework. Among all of the considered interpolation methods, Linear and IDW indicated the lowest mean error ranging from 4.3 to $8 \mathrm{~dB}$. However, it should be noted that all the above research works concentrated on the signal coverage prediction incorporating a single BS. Therefore, those results are not directly comparable to the results of our study, since interpolating a multi-BS coverage map is a challenging and complex task.

\section{Coverage Assessment Metrics}

When conducting a coverage assessment of radio network deployments, one needs to define a coverage metric. Generally, coverage quality indicators can be separated into two main groups, namely, averaged and cumulative parameters. As an example in the first group, the authors in [22] utilized the ratio between misclassified regions and the total area as their performance indicator. Even though this parameter allows for simple comparison of the results via a single variable, it does not provide any measure for comparing the predicted signal level accuracy. Instead, it rather focuses on the borderline values denoting the covered area.

In [26], the target metric was defined as cumulative probability of the RSS error. On top of that, the mean error as a function of the removed fingerprints was used for an initial verification of the predicted values. This metric is similar to that utilized in our work but it does not consider 
positive and negative deviations by using modulus. Finally, in [25], the authors defined their parameter of interest as cumulative density function (CDF) of empirical errors between the modeled and the predicted values. Such a metric offers useful information about the distribution of error. However, it does not allow for simple comparison of deviations as a single variable.

In our work, we combine the two approaches employed in research works [25] and [26]. However, in contrast to the metric given by [25], we utilize the part of the area covered with a signal level of at least $x \mathrm{dBm}$. Aside from the predicted values, it also includes the results given by the reference model. To provide an assessment metric permitting for a single-number comparison of the coverage accuracy, we further incorporate the parameter inspired by [26]. In contrast to the formula in [26], our performance indicator includes a modulus operation to account for positive as well as negative deviations.

\section{Our MEASUREMEnt CAMPAigN}

To obtain the essential input data, we carried out a widescale measurement campaign in the city of Brno, the secondlargest city of the Czech Republic. The measurement results were collected during the eight-month period between February and September 2019. The subject campaign covered over 300 unique measurement locations next to the stop stations of public transport lines (i.e., buses, trams, and trolleybuses) 2 . The locations of the measurement points were selected to reflect one of the potential use cases of LPWAN technologies, which is localization services in Smart City applications [27].

In what follows, we offer a brief description of the selected LPWAN technologies, the measurement equipment, and the operating environment.

\section{A. LPWAN Technologies}

For our measurement campaign, we selected the three major LPWAN technologies (Sigfox, LoRaWAN, and NB-IoT) that are publicly available to the end-customers in the Czech Republic. The first two representatives, Sigfox and LoRaWAN, belong to a large group of solutions operating in the licenseexempt industrial, scientific, and medical (ISM) band, see Tab. I]. Conversely, NB-IoT is one of the CIoT technologies operating in the licensed LTE band. The government regulates the utilization of both frequency ranges (in the Czech Republic, Czech Telecommunication Office is the regulator). However, the ISM conditions are much more stringent as compared with the licensed case.

1) Sigfox: This ultra-narrowband technology is considered to be the first publicly available LPWAN solution with commercial roll-outs in 2012. The network infrastructure consists of end devices (EDs), gateways (GWs), and the cloud core. The data is transferred wirelessly to the GW and then conveyed to the cloud system via the Internet connection. The transmission of data is always initiated by the ED and can commence

\footnotetext{
${ }^{2}$ The map of the measurement points in question is accessible online via Google Maps
}

TABLE I: Key parameters of LPWAN technologies.

\begin{tabular}{|c|c|c|c|}
\hline & LoRaWAN & Sigfox & NB-IoT \\
\hline Coverage (MCL) & $157 \mathrm{~dB}$ & $162 \mathrm{~dB}$ & $164 \mathrm{~dB}$ \\
\hline Technology & $\begin{array}{c}\text { PHY - Proprietary } \\
\text { MAC - Open }\end{array}$ & Proprietary & Open LTE \\
\hline Spectrum & Unlicensed & Unlicensed & Licensed \\
\hline Duty cycle limitation & Yes & Yes & No \\
\hline Max. EIRP & $\begin{array}{c}16 \mathrm{dBm} \\
(40 \mathrm{~mW}) \\
\end{array}$ & $\begin{array}{l}16 \mathrm{dBm} \\
(40 \mathrm{~mW}) \\
\end{array}$ & $\begin{array}{c}23 \mathrm{dBm} \\
(200 \mathrm{~mW}) \\
\end{array}$ \\
\hline Modulation & $\begin{array}{c}\text { Lora (CSS), } \\
\text { FSK }\end{array}$ & $\begin{array}{l}\text { D-BPSK (UL), } \\
\text { GFSK (DL) }\end{array}$ & $\begin{array}{l}\pi / 2 \text {-BPSK, } \pi / 4 \text {-QPSK, } \\
\text { QPSK (DL }+ \text { multi-tone) }\end{array}$ \\
\hline Data rate in DL & $\begin{array}{l}0.25-11 \text { kbps (LoRa) } \\
50 \text { kbps (FSK) }\end{array}$ & $0.6 \mathrm{kbps}$ & $0.5-27.2 \mathrm{kbps}^{1}$ \\
\hline Data rate in UL & $\begin{array}{l}0.25-11 \text { kbps (LoRa) } \\
50 \text { kbps (FSK) }\end{array}$ & $0.1 \mathrm{kbps}$ & $0.3-62.5 \mathrm{kbps}^{1,2}$ \\
\hline Max. UL payload & $242 \mathrm{~B}$ & $12 \mathrm{~B}$ & $1600 \mathrm{~B}^{1}$ \\
\hline Max. DL payload & $242 \mathrm{~B}$ & $8 \mathrm{~B}$ & $1600 \mathrm{~B}^{1}$ \\
\hline TX current & $45 \mathrm{~mA}$ & $55 \mathrm{~mA}$ & $220 \mathrm{~mA}$ \\
\hline Sleep current & $<2 \mathrm{uA}$ & $<2 \mathrm{uA}$ & $<3 \mathrm{uA}$ \\
\hline Battery life & $10+$ years & $10+$ years & $10+$ years \\
\hline Module cost & $6 \$$ & $2 \$$ & $8 \$$ \\
\hline Security & $\begin{array}{c}\text { Medium } \\
\text { AES-128 }\end{array}$ & $\begin{array}{c}\text { Low }^{3} \\
\text { AES-128 }\end{array}$ & $\begin{array}{c}\text { Very high } \\
\text { 3GPP (128-256 bit) }\end{array}$ \\
\hline
\end{tabular}

${ }^{1}$ The value is release-dependent (Rel. 13)

${ }^{2}$ Valid for multi-tone transmission.

${ }^{3}$ By default, encryption is not active.

at any time. However, the devices cannot violate the limitation on the radio channel utilization imposed by the respective authorities. With that in mind, the uplink transmission is constrained to 140 messages per day, with the maximum size of 12 bytes. Limitations in the downlink channel are even more strict with only 4 messages per day having the payload of 8 bytes.

The uplink transmission is modulated with differential binary phase-shift keying (D-BPSK) having the rate of $100 \mathrm{bps}$ (this value is valid for radio configuration (RC) 1 in EU, and may differ in other regions). The carrier frequency is selected randomly, typically within the $200 \mathrm{kHz}$ band. Center frequency in $\mathrm{RC} 1$ is set to $868.13 \mathrm{MHz}$ with the maximum effective isotropic radiated power (EIRP) of $16 \mathrm{dBm}$. The reliability of data transfer is ensured by two mechanisms: (i) repetition and (ii) spatial diversity. Message transmission is repeated three times, each one employing random carrier frequency selection. Further, ED is not attached to a particular BS, but its data is instead received and forwarded by any of the reachable GWs [1].

2) LoRaWAN: The second well-known representative of the LPWAN family operating in the ISM band is LoRaWAN. Its standard network structure is similar to that of Sigfox by comprising EDs, at least one GW, and a network server (NS). In addition, the system may feature a specialized network join (NJ) server to handle roaming between networks. In most cases, a transmission is initiated by the ED using an Aloha-like channel access mechanism [28]. It allows the ED to initiate communication at any time by not violating the operational restrictions on the selected radio channel. Within the EU region, the ED selects one of up to sixteen available channels in the frequency range from 863 to $870 \mathrm{MHz}$ with the bandwidth of 125 or $250 \mathrm{kHz}$. The first three channels (868.1, 868.3 , and $868.5 \mathrm{MHz}$ ) must be supported by every ED and cannot be changed. 
The ISM frequency band of $868 \mathrm{MHz}$ imposes the limitation of $1 \%$ duty cycle with the maximum EIRP of $16 \mathrm{dBm}$. At the physical layer, the data is transferred with a proprietary long-range (LoRa) modulation based on the spread spectrum technique named chirp spread spectrum (CSS). This mechanism permits LoRaWAN to operate below the noise floor. The LoRa modulation rate can be adjusted by the spreading factor (SF) parameter, which can vary from 7 to 12 . The $\mathrm{SF}$ value controls the modulation robustness, thus directly affecting radio coverage. The achievable bitrate in the $125 \mathrm{kHz}$ channel therefore varies from 250 (SF 12) to 5470 (SF 7) bps, which results in the maximum payload size of 51 (SF 12) up to 242 (SF 7) bytes. Due to power efficiency considerations, the static EDs are commonly configured to use the lowest SF that allows for reliable communication [2].

3) NB-IoT: Unlike the two LPWAN options described above, NB-IoT relates to the cellular technology operating in the licensed bands. It was introduced in 2016 as part of the 3GPP Rel. 13 with the first commercial roll-outs in the following year [29]. The system is composed of user equipment (UE), evolved Node B (eNodeB), evolved packet core (EPC), and application servers. As the terminology suggests, NB-IoT reuses a significant fraction of the existing LTE infrastructure. In most cases, the LTE system can be upgraded to support the latest specifications via a software update [29], [30]. As compared to LTE, the bandwidth of the NB-IoT system is reduced to $180 \mathrm{kHz}$; thus, it can be deployed within a single physical resource block (PRB). On top of that, NB-IoT can operate in a stand-alone mode (single global system for mobile communications (GSM) carrier) or in a guard band of the LTE system [30].

In contrast to LoRaWAN and Sigfox, NB-IoT uplink operation is not based on pure Aloha but utilizes its slotted version for channel access and then resorts to using the time-frequency resources allocated by an evolved NodeB (eNodeB) [28]. Since NB-IoT uses licensed frequency bands (predominantly, sub-GHz spectrum), there are no duty-cycle restrictions. The maximum uplink payload at the physical layer is 1000 bits (up to 2536 bits in Rel. 14) due to limitations on the transport block size (TBS). Further, the packet data convergence protocol (PDCP) layer permits the protocol data units (PDUs) with the size of up to 1600 bytes. The transmit power of the $\mathrm{UE}$ can be as high as $23 \mathrm{dBm}$ (there is additional support for $20 \mathrm{dBm}$ and $14 \mathrm{dBm}$ power classes). In the case of a single tone uplink transmission, NB-IoT supports 15 and $3.75 \mathrm{kHz}$ subcarrier spacing with the single carrier-frequency division multiple access (SC-FDMA).

The uplink data is modulated by utilizing binary-phase shift keying $(\pi / 2$-BPSK) or quadrature phase-shift keying $(\pi / 4$ QPSK) with the continuous phase to reduce peak-to-average power ratio (PAPR). However, the highest bitrate can only be achieved with multi-tone transmissions utilizing QPSK modulation. If all the twelve tones are used, the theoretical throughput can be as high as $62.5 \mathrm{kbps}$ (up to $159 \mathrm{kbps}$ in Rel. 14). In the downlink, NB-IoT supports only $15 \mathrm{kHz}$ subcarrier spacing with orthogonal frequency division multiple access
(OFDMA). Further, the modulation scheme is limited to QPSK with the maximum TBS of 680 bits (up to 2536 bits in Rel. 14), which results in the maximum data rate of $27.2 \mathrm{kbps}$ (up to $127 \mathrm{kbps}$ in Rel. 14) [30], [3]. The extended coverage (+20 dB w.r.t. LTE) is achieved primarily via repetitions. The random access channel procedure and all uplink transmissions may benefit from up to 128 repetitions [30].

\section{B. Measurement Equipment and Setup}

During our Sigfox and LoRaWAN measurements, we utilized field testers by the company Adenuis. Specifically, we used ARF8121AA for Sigfox and ARF8123AA for LoRWAN. Both devices are equipped with integrated $0 \mathrm{dBi}$ omnidirectional antenna and offer the theoretical communication range of $15 \mathrm{~km}$. The effective radiated power (ERP) in both cases was set to $14 \mathrm{dBm}(25 \mathrm{~mW})$. When configured with SF 12 and the coding rate of $4 / 5$, this allows to achieve the sensitivity of $-137 \mathrm{dBm}$ for the LoRaWAN technology. The utilized coding rate does not offer the longest communication range, but it was selected due to the requirement imposed by the LoRaWAN regional specifications [31]. For Sigfox, the standard BS SBST3 provides the sensitivity of up to $-146 \mathrm{dBm}$ [32].

In the case of NB-IoT measurements, we employed our own testing device developed at Brno University of Technology (BUT). The measurement unit is equipped with SARA N210 NB-IoT module by the company uBlox. The selected module implements the NB-IoT specifications according to Rel. 13 with the maximum transmit power of $23 \mathrm{dBm}(200 \mathrm{~mW})$, thus providing the sensitivity of up to $-135 \mathrm{dBm}$. The said module incorporates only one frequency band, i.e., B20 $(800 \mathrm{MHz}$, uplink (832 - $862 \mathrm{MHz})$, downlink (791 - $821 \mathrm{MHz})$ ), which is utilized by the Vodafone's NB-IoT network in the Czech Republic as the main frequency band [33]. On top of that, radio wave propagation is facilitated by the omnidirectional half-wave antenna with $2 \mathrm{dBi}$ gain. Specifically, it is a pentaband antenna by RF Solutions that is designated as ANT-PCB8121-FL [34].



Fig. 1: Network architecture of selected LPWAN standards.

For all the measurement points, the test devices were deployed at a selected location and positioned approximately one meter above the ground level away from buildings and other obstacles. Then, the testing units were powered up to make them transmit ten messages with the period of $30 \mathrm{~s}$. To 
avoid interference between the LPWAN technologies, the measurement equipment was utilized sequentially. The illustrative path of a datagram for each technology is depicted in Fig.1 The message size was set to 12 bytes for all the considered radio technologies, to remain in-line with the limitations of the Sigfox operation, see Section III-A1, The time frames and the locations of each measurement point were recorded for further statistical analysis. The preliminary positions of the measurement points were acquired from the map and verified by Adenuis testers having a built-in global navigation satellite system (GNSS) receiver.

Once the field measurements were completed, all of the available data were collected from the appropriate web management interfaces. At the time of conducting the measurements and processing the data, only the locations of the LoRaWAN GWs were available publicly and accessible via the management interface. To acquire the locations of Sigfox and NB-IoT BSs, we had to contact the network operators Simplecell (Sigfox) and Vodafone Czech Republic (NB-IoT). In accordance with the effective agreements, the precise locations of the BSs are known to the authors and have been employed in our analysis, but they cannot be revealed publicly.

\section{Data Pre-processing}

Since the NB-IoT module is always connected to a single $\mathrm{BS}$, the respective data processing was a straightforward procedure. All the signal quality indicators received from a certain test location were averaged to obtain a single RSRP value, which served as an input to the subsequent interpolation methods. In the cases of Sigfox and LoRaWAN, the situation was different. The devices operating under these two technologies are not connected to a specific GW, and their messages are received by each available $\mathrm{BS}$ within the communication range.

In the extreme case, we observed a single packet being received by 15 LoRaWAN GWs. To acquire a single value, we selected the GW with the best average RSSI and used this result for the following interpolation methods. The points in outage were excluded from the consideration. In the last step, we used the haversine formula to obtain the distances between each measurement point and the corresponding BS/GW.

\section{Measurement Environment and Network Configurations}

Our measurement campaigns were executed around the city of Brno at 303 unique locations spanning over $12 \mathrm{~km}$ north to south and $24 \mathrm{~km}$ east to west. The results of these coverage measurements for each of the three LPWAN technologies are depicted in Fig. 2] As an indicator of the coverage quality at a certain location, we utilized the RSSI values for Sigfox and LoRaWAN. Conversely, for NB-IoT, RSRP served as the primary signal quality indicator. It provides a more accurate assessment of radio channel conditions by measuring only the R0 (also R1, if available) reference signal, thus excluding the interference from other antenna sectors and unrelated channels [30]. Furthermore, RSRP is not technically available for LoRaWAN and Sigfox as these much simpler technologies do not utilize multi-sector antennas and reserved reference signal channels. For these reasons, the signal strength metric is limited only to RSSI, which derives the received power over the entire bandwidth.

Summarizing the processed data, all three technologies offered relatively reliable service, with only a few measurement points being in outage. The most robust connectivity was provided by the NB-IoT network - with only three unserved points - followed by Sigfox and LoRaWAN with ten and sixteen outage cases, respectively. However, these differences are marginal for the scale in question. More significant differences become visible when the signal levels are examined. For NBIoT, the average value of RSRP was $-76 \mathrm{dBm}$, whereas the mean signal levels for Sigfox and LoRaWAN were close to $-100 \mathrm{dBm}$. More specifically, the values were $-112 \mathrm{dBm}$ for Sigfox and $-98 \mathrm{dBm}$ for LoRaWAN.

These differences are attributed to the features of the technologies and the densities of the BSs. With respect to the latter, the numbers of NB-IoT BSs in our test area greatly exceeded those for the two counterpart solutions. Specifically, NB-IoT is in the first place with 78 dedicated BSs having more than a quadruple volume of GWs belonging to other LPWAN solutions. In contrast, Sigfox and LoRaWAN networks only feature 13 and $19 \mathrm{GWs}$, respectively. The actual BS density also impacts the distances between the measurement points and their closest GW/BS. For NB-IoT, the average distance to the nearest BS did not exceed $0.52 \mathrm{~km}$, whereas for Sigfox it was $3.45 \mathrm{~km}$ and $1.86 \mathrm{~km}$ in the case of LoRaWAN.

\section{Proposed Methodology}

In this section, we first outline the proposed methodology for identifying the minimum set of measurement points. Then, we proceed by specifying the reference model employed by our study as well as the considered interpolation and thinning algorithms.

\section{A. Procedure and Metrics of Interest}

The proposed procedure for identifying the minimum set of measurement points to assess the network coverage can be divided into several steps, as depicted in Fig. 3 In the first one, we utilize the knowledge of the BS locations across the area of interest, and combine it with standardized propagation models to construct a reference model for each LPWAN technology.

At the second stage, we build a model without explicit knowledge of the BS locations by employing interpolation techniques to infer the RSRP (or RSSI for LoRaWAN and Sigfox) values at each point inside the considered area. To identify the minimum set of measurement points required for accurate coverage assessment, we apply and study two fundamentally different reducing techniques, which are probabilistic and deterministic thinning.

We expect that these algorithms will significantly impact the quality of hypothesis since random selection of measurement points should provide inferior accuracy as compared to more complex deterministic selection. Hence, we consider a range 


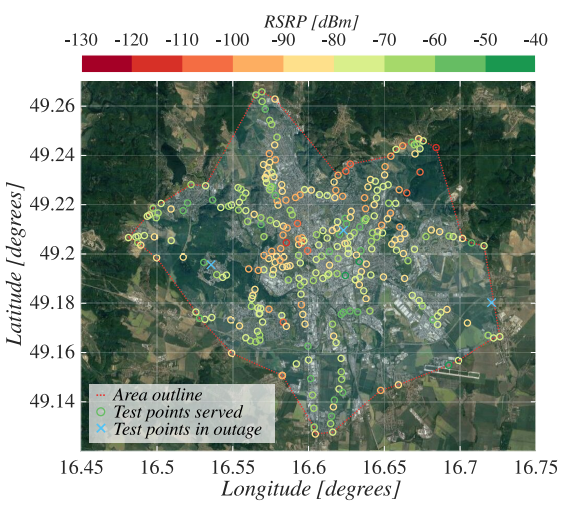

(a) NB-IoT

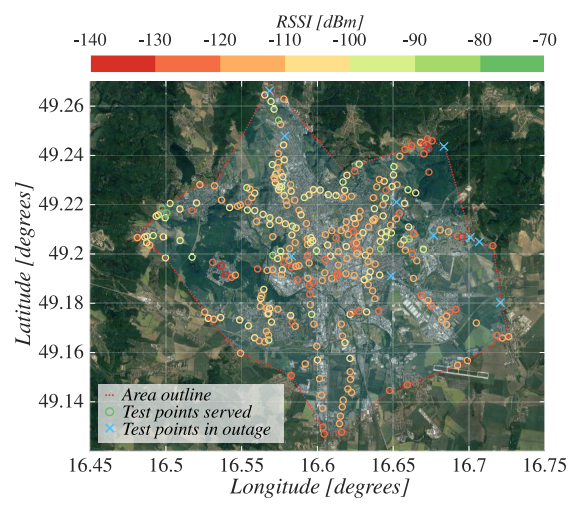

(b) Sigfox

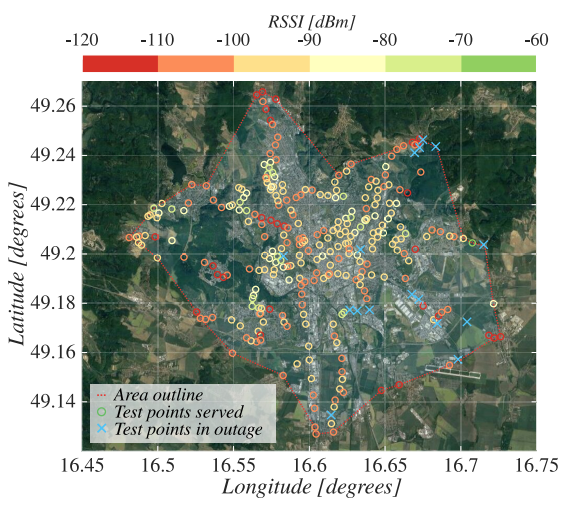

(c) LoRaWAN

Fig. 2: Coverage of LPWAN technologies in the city of Brno.

Reference model

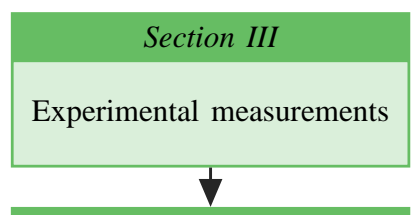

Section $I V-B I$

Derivation of the reference propagation model based on empirical measurements

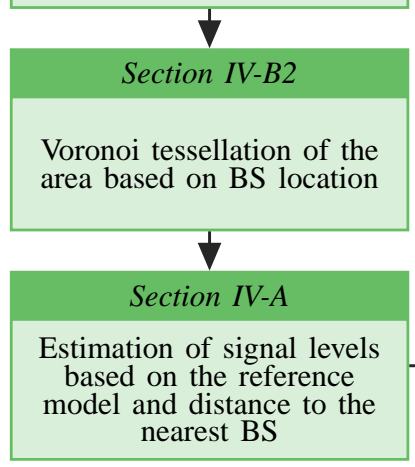

Fig. 3: Main steps of our assessment process.

of parameters for these thinning approaches. In Section $\mathrm{V}$ we also identify which one demonstrates the best performance.

For both thinning algorithms, we consecutively reduce the number of retained points. Further, we apply the metrics defined below to quantitatively characterize the distance between the data set produced by the reference model (derived with the knowledge of BS locations) and the one with the reduced number of measurement points (created without the knowledge of BS locations). Finally, we compare the prediction accuracy of the reference model and the interpolation methods against the measured values. Based on these results, we make conclusions on the minimum number of measurement points needed to provide the coverage quality assessment with a given maximum deviation from the measured values.

As a coverage assessment parameter, we employ the proba- bility that a certain fraction of area is covered with the signal level of at least $x \mathrm{dBm}$. In the sequel, we estimate this metric for both the reference model and the interpolated data by using the following approach. A regular grid with the uniform step is applied to the area of interest. The coverage quality is then estimated by using the sample values of RSRP/RSSI at each of those points. As one may observe, this parameter can be interpreted as an inverse CDF of the RSRP/RSSI values.

Note that the introduced metric allows for a convenient visual comparison between the reference model and the proposed option. However, it does not permit to quantify the distance between the two given models. To facilitate a quantitative comparison between the models, we propose the use of the mean absolute error (MAE) metric that specifies the cumulative deviation between any two models under consideration. We define this parameter as follows

$$
Q=\frac{1}{N} \sum_{i=1}^{N}\left|R_{i}^{(1)}-R_{i}^{(2)}\right|,
$$

where $R_{i}^{(1)}$ and $R_{i}^{(2)}$ are the sample values of the two models at the same point and $N$ is the total number of points on the interpolation grid. Note that the modulus is used here to account for positive and negative deviations. Each point $i$ represents one cell of the regular grid with the resolution of $50 \mathrm{~m}$ outlined by the area of the test locations as depicted in Fig. 2 These cells serve as an output data set of the interpolation algorithm, which estimates the RSRP/RSSI value for each point $i$.

As one may observe, the averaged integral metric specified in (1) is independent of the number of points $N$ where the coverage metric is evaluated and produces the absolute deviation from the actual coverage averaged over the total number of interpolated points $N$.

\section{B. Reference Model}

We proceed by defining the baseline reference models for each of the considered LPWAN technologies. A reference model is intended to compare the widely used radio propagation models with the interpolation methods in terms of the 


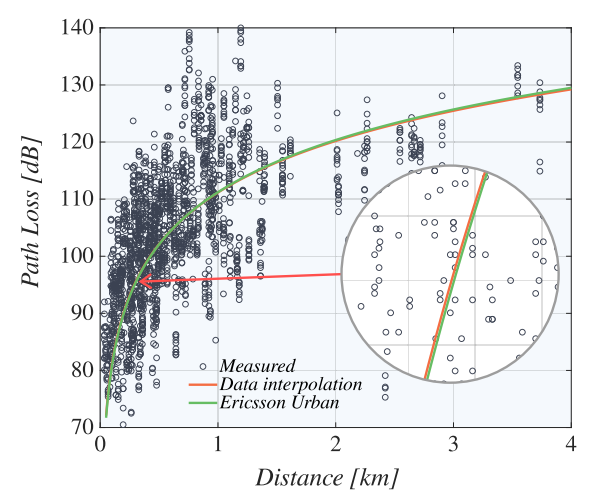

(a) NB-IoT

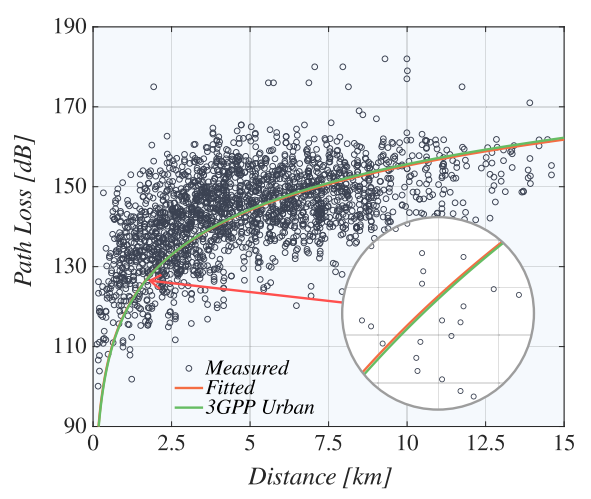

(b) Sigfox

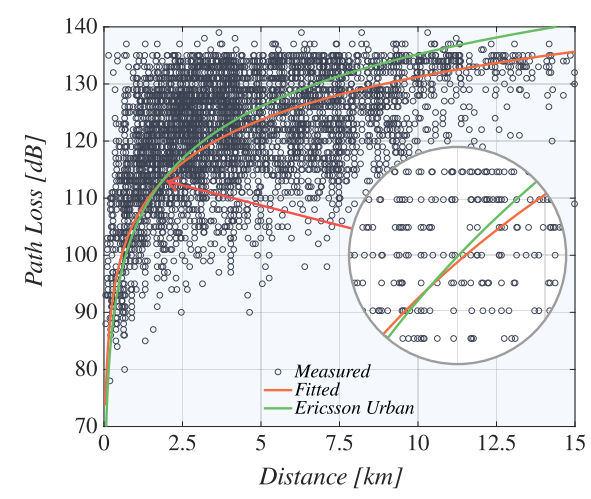

(c) LoRaWAN

Fig. 4: Fitting of propagation model to measurement data.

prediction accuracy. Each reference model assumes that the exact BS locations are available.

1) Reference Path Loss Model: At the first step of our modeling, we employ the measured signal levels at each location to derive the respective path loss (PL) value as

$$
P L=\left|P_{R X}\right|+S N R+P_{T X}+G ; \quad P_{R X} \leq 0,
$$

where $\left|P_{R X}\right|$ denotes the absolute value of the received signal power in $\mathrm{dBm}$ (RSRP in the case of NB-IoT; RSSI for Sigfox and LoRaWAN), SNR is the signal-to-noise ratio, $P_{T X}$ represents the transmitter's effective radiated power, and $G$ stands for the sum of antenna gains.

To derive the continuous path loss curve from the discrete measurement samples, we utilize a non-linear regression to obtain the expected coefficients of the log-distance PL model [15] as defined by

$$
P L=\overline{P L}\left(d_{0}\right)+10 \gamma \log _{10}\left(\frac{d}{d_{0}}\right) .
$$

In the above, $\overline{P L}\left(d_{0}\right)$ represents the PL at the reference distance $d_{0}=0.1 \mathrm{~km}, \gamma$ is the PL exponent, and $d$ denotes the distance between the end device and the BS. The floating intercept value $\overline{P L}\left(d_{0}\right)$ is calculated from the free-space path loss formula with an additional $10 \mathrm{~dB}$ attenuation capturing the propagation losses in urban environment. The resulting fitted models together with the measured data are depicted in Fig. 4.

These empirical models derived from the measurements data characterize the city of Brno, but their accuracy may be questionable for other locations and thus needs to be validated. Therefore, at the second step, we select a validated PL model representing the closest match to our empirical model. During our analysis [35] of the propagation models available for LPWAN technologies, e.g., Okumura-Hata, Ericsson 9999, Stanford University Interim, and COST (European Cooperation in Science and Technology) 231 WalfischIkegami, we found out that none of them provide sufficient accuracy. Notably, the selected models in their basic form significantly overestimate the path loss values for all the considered LPWAN technologies.
Therefore, we decided to utilize the propagation models from our previous work, representing the aforementioned options fine-tuned for particular communication technologies. For our approximation, we assumed $1.2 \mathrm{~m}$ ED height with the BS antenna $15 \mathrm{~m}$ above the rooftop level of $30 \mathrm{~m}$ and the carrier frequency of $861.7 \mathrm{MHz}$. The latter value is valid for uplink NB-IoT operation in the guard-band mode within band $20(800 \mathrm{MHz})$, which is utilized by Vodafone's network in the Czech Republic. For other LPWAN technologies, we imply the frequency of $868.1 \mathrm{MHz}$. This value is close to the center uplink frequency used by Sigfox and corresponds to the first channel of LoRaWAN.

The subject fine-tuned models were cross-validated with the measurement data in two cities. Hence, it is expected that their accuracy will be sufficient in the case of a typical midsize European town. As it is depicted in Fig. 4, both NBIoT and LoRaWAN are most accurately represented by the Ericsson Urban model, whereas 3GPP Urban captures Sigfox the best. The parameters of each propagation model are given in Table II

TABLE II: Propagation model parameters [35].

\begin{tabular}{|c|c|c|c|}
\hline Technology & Model & $\overline{P L}\left(d_{0}\right)$ & $\gamma$ \\
\hline NB-IoT & Ericsson Urban & 111.21 & 3.04 \\
\hline Sigfox & 3GPP Urban & 118.04 & 3.76 \\
\hline LoRaWAN & Ericsson Urban & 104.82 & 3.04 \\
\hline
\end{tabular}

Both models are best suited for urban and suburban environments outside the high-rise core, where the buildings have a near-uniform height. This description characterizes the city of Brno well, as its urban layout primarily comprises of midrise buildings. More precisely, the Ericsson Urban model is applicable for frequencies up to $1900 \mathrm{MHz}$, BS heights ranging from 20 to $200 \mathrm{~m}$, ED heights between 1 and $5 \mathrm{~m}$, with BSED separation distances up to $100 \mathrm{~km}$. The $3 \mathrm{GPP}$ Urban model can be used for frequencies of up to $2600 \mathrm{MHz}$, with a cell radius less than $8 \mathrm{~km}$ and BS antenna height of up to $50 \mathrm{~m}$ above the average rooftop level [36].

2) Voronoi Tessellation: The next step of the reference model derivation concerns the Voronoi tessellation. Its primary 
purpose is to divide the area covered by the BSs into the corresponding regions that consist of all the points on the plane closer to the seed (BS) than to any other. In other words, Voronoi tessellation dissects the area into polygons with the BS located inside representing the closest BS for all the points in that polygon. The outcome of the Voronoi tessellation process for NB-IoT technology is depicted in Fig. 5. A similar tessellation procedure is repeated for Sigfox and LoRaWAN BS locations.

Finally, the radio propagation models derived in the previous step are applied to the tessellated area to specify the expected signal level at each point of interest. These points are defined by a regular grid with $50 \mathrm{~m}$ resolution, thus creating the baseline coverage models. In what follows, these constructs are named the "reference" models. It is worth noting that these reference models have been derived with the exact knowledge of BS locations and then compared against the models generated using the interpolation methods, which were constructed using only the measurement data.

At this point, it is crucial to highlight that the reference model was not used to exclusively quantify the accuracy of the results. We do not consider the constructed reference model to be the so-called "ground truth" for a comparison with the proposed interpolations-based approach. Therefore, the presented results should be considered as a comparison of the two models rather than a comparison of the "ground truth" with the results of the proposed methodology.

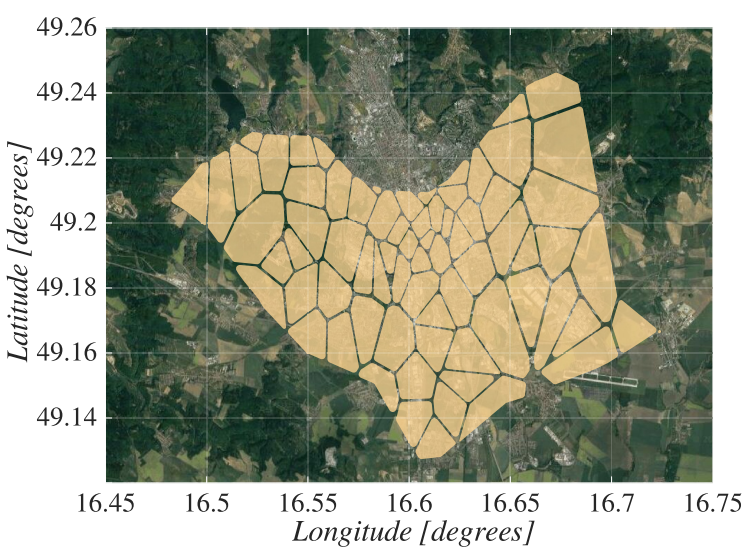

Fig. 5: Voronoi tessellation of NB-IoT deployment.

\section{Interpolation Algorithms}

Fig. 2 indicates that our measurement data does not spread densely across the entire area. Therefore, to predict the service quality at all the points of the regular grid, one may resort to the use of interpolation methods. Since the measurement locations are not spread evenly, we exclusively consider the interpolation methods for scattered data. In our work, we select five most common representatives that can interpolate the arbitrarily spaced data. The chosen algorithms cover the entire spectrum of complexity from simpler and more straightforward, such as (i) Nearest-neighbor interpolation, to the schemes with intermediate complexity, including (ii)
Linear, (iii) Natural-neighbor, and (iv) IDW approaches, to more computationally demanding, e.g., (v) Kriging algorithm.

1) Nearest-neighbor: This method is a simple approach to data interpolation. The initial step is Delaunay triangulation of known points $x$. Then, the vertices are lifted by the magnitude of $V$ into the dimension orthogonal to $x$. The same lifting procedure is also conducted for the requested point $x_{q}$. In general, this involves traversing the triangulation data structure to find the triangle enclosing the requested point. Once that position is established, the closest known point value is reused in the requested point $x_{q}$ [37], [38].

The main advantage of this method is its simplicity and low computational cost. On the other hand, the resultant interpolation does not create new values for the requested points but rather duplicates the existing ones. This leads to formation of sharp transitions at the edges of the neighboring cells [38].

2) Linear: The main idea of Linear interpolation is equivalent to that of the Nearest-neighbor method [37]. The area defined by the measurement points is tessellated with Delaunay triangulation, and the vertices are lifted orthogonally to the known points $x$ by the magnitude of $V$. However, the value of the requested point $x_{q}$ is not duplicated from the closest known point but is rather calculated as the weighted sum of the magnitudes of the three vertices that constitute the enclosing triangle.

Linear interpolation utilizes the Barycentric coordinate system [39] to overcome the problem of value prediction where the points lie directly on the connecting lines of vertices. If the requested point $x_{q}$ is placed inside the triangle and is connected to each vertex, the resulting diagram consists of three separate triangles with the areas of $A_{1}, A_{2}$, and $A_{3}$. If $A$ denotes the area of the original triangle, one can calculate the Barycentric coordinates $\alpha, \beta$, and $\gamma[40]$ as

$$
\alpha=\frac{A_{1}}{A}, \quad \beta=\frac{A_{2}}{A}, \quad \gamma=\frac{A_{3}}{A} .
$$

The sum of these coordinates is always equal to one; therefore, it is possible to calculate the third coordinate by subtracting the sum of the first two from unity. The resulting value of the interpolated point $x_{q}$ is produced as

$$
x_{q}=\alpha V_{A}+\beta V_{B}+\gamma V_{C}
$$

where $V_{A}, V_{B}$, and $V_{C}$ represent the vertices of the triangle.

The main benefit of this interpolation method is its speed (considerably slower than Nearest-neighbor though) and ease of implementation. However, Linear interpolation only provides $C^{0}$ smoothness [38].

3) Natural-neighbor: The essence of Natural-neighbor interpolation is a Voronoi diagram structure. In the initial step, known points are used to tessellate the covered area. Each cell of such a diagram contains one generating sample point that defines the value for the entire cell. Voronoi tessellation is discontinuous by its nature, but it has useful characteristics that Natural-neighbor interpolation utilizes. Here, each edge represents a link between two generating sample points. This 
property is used to select the corresponding neighbors for the interpolation [41].

The next step in the interpolation process is point insertion. The requested point $x_{q}$ is inserted into the Voronoi diagram, and a new polygon generated by the requested point $x_{q}$ is added to the structure. Consequently, part of the area from the neighboring cells is transferred to thus inserted polygon, and some edges covered by the new cell are subsumed. The main goal of the next step is to identify the weights of the neighboring samples and combine them with the sample values to obtain an estimate of the requested point $x_{q}$. Hence, each of the neighboring cells contributes a certain fraction of its area to the new cell.

Further, the value of the requested point $x_{q}$ is computed as the weighted average of the surrounding sample polygons. This approach is similar to that in Linear interpolation but the mean is not limited to three vertices of the enclosing triangle. The fractional contribution of each polygon $\lambda_{i}$ can be written as

$$
\lambda_{i}=\frac{A_{i}}{A},
$$

where $A_{i}$ is the area of the contributing cell $i$ and $A$ is the total area of the inserted polygon. The sum of these weights is always one. Finally, the interpolated value is calculated as

$$
x_{q}=\sum_{i=0}^{N} \lambda_{i} \cdot z_{i}
$$

where $N$ is the number of neighbors surrounding the inserted polygon, $\lambda_{i}$ is the weight of each element, see (6), and $z_{i}$ is the value of the sample point [42].

The key advantages of this interpolation method lie in its relative simplicity, speed, and resilience to biases introduced by how the samples may cluster. One of the drawbacks though is the slope discontinuity; therefore, it cannot offer $C^{1}$ continuity over the entire surface [41], [42].

4) $I D W$ : The main premise of IDW interpolation is in that the values of more proximate points are related closer than for the farther away ones. In other words, the interpolated values are calculated by using a linearly weighted combination of the sample point sets. The weight is defined as an inverse function of distance. The value of the requested point $x_{q}$ is calculated as

$$
x_{q}=\frac{\sum_{i=o}^{n} d_{i}^{-p} \cdot z_{i}}{\sum_{i=0}^{n} d_{i}^{-p}},
$$

where $z_{i}$ is the value of the sample point, $d_{i}$ is the distance between the requested and the sample points, and $p$ is the power parameter [43]. The latter controls the significance of the known point $x$ for the interpolated value based on its distance to the requested point $x_{q}$.

Hence, the closest points have the most significance for the resulting value of the requested point. The power value is not defined strictly and does not have connection to the real-world process. The typical convention here is to use the power of two, which we also employed in this work. Finally, the value of $n$ determines the number of samples for interpolation and may be up to the total number of points in the data set (typically three or more though).

The selection of input sample points significantly impacts the behavior of the interpolated surface. Along these lines, IDW offers immense variability as it can use, e.g., a fixed search radius where the requested value is calculated from the points at the pre-set maximum distance. Further, the shape of the search window does not have to be a circle. Another option is variable radius where the search window is defined by the number of neighboring points used for calculation. In this work, we follow the latter approach, where the requested value $x_{q}$ is derived from the five closest points [44].

IDW is an intuitive, easy to understand, and computationally efficient interpolation method. However, its accuracy decreases if the sample points are not distributed evenly. Also, the resulting surface does not provide $C^{1}$ smoothness. Moreover, the selection of input parameters is entirely arbitrary but it influences the resulting interpolation surface drastically [43].

5) Kriging: This interpolation algorithm belongs to the class of geostatistical methods based on statistical models that incorporate autocorrelation (in the sense of mathematical relationship between the measured points). Hence, geostatistical techniques have the capability of producing a prediction surface, and also offer an assessment of the interpolation accuracy. Kriging accuracy is the highest when there is spatially correlated distance or directional bias in the input data [45].

Producing interpolated values is similar to how it is done by Natural-neighbor or IDW according to (7). However, the process of weight derivation differs drastically. Kriging weights are based not only on the distance to the sample point but also on the overall spatial arrangement of the measurement points. To achieve this, Kriging follows a two-step process as it (i) creates a covariogram and covariance functions, as well as (ii) predicts unknown values.

The initial step of the Kriging procedure is in designing an experimental semi-variogram [46], which is given as

$$
\gamma(h)=\frac{1}{2 N(h)} \sum_{i=1}^{N(h)}\left[z\left(x_{i}\right)-z\left(x_{i}+h\right)\right]^{2},
$$

where $N(h)$ is the number of pairs that are separated by the distance of $h$ and $z\left(x_{i}\right)$ is the value of the input point. The experimental semi-variogram is then fitted to the empirical semi-variogram. This step is needed because the experimental semi-variogram is not a continuous function but is rather represented by a set of points. For our work, we employ Spherical semi-variogram, which is defined as

$$
\gamma(h)=\left\{\begin{array}{ll}
c_{o}+c_{1}\left[\frac{3 h}{2 a}-\frac{1}{2}\left(\frac{h}{a}\right)^{3}\right] & \text { for } 0<h<a \\
c_{o}+c_{1} & \text { for } h \geq a
\end{array},\right.
$$

where $a$ is the range, $c_{0}$ is the nugget variance, and $c_{0}+c_{1}$ is the sill. Selection of semi-variogram impacts prediction of unknown values. Therefore, it is necessary to choose a model 
that fits sample data the best. The last step of the Kriging process is prediction of the requested points. In our work, we use Ordinary Kriging but there are other variants of the prediction process [47]. For Ordinary Kriging, the system is produced as

$$
\left[\begin{array}{c}
\lambda_{1} \\
\vdots \\
\lambda_{n} \\
\mu
\end{array}\right]=\left[\begin{array}{cccc}
C_{11} & \cdots & C_{1 n} & 1 \\
\vdots & \ddots & \vdots & \vdots \\
C_{n 1} & \cdots & C_{n n} & 1 \\
1 & \cdots & 1 & 0
\end{array}\right]^{-1}\left[\begin{array}{c}
C_{10} \\
\vdots \\
C_{n 0} \\
1
\end{array}\right]
$$

where $\mu$ is the Lagrange parameter and $C_{1 n}$ is the covariance between the locations of sample points $x_{1}$ and $x_{n}$. The latter is computed as

$$
C_{1 n}=\operatorname{Cov}\left(x_{1}-x_{n}\right)=C(0)-\gamma\left(x_{1}-x_{n}\right),
$$

where $C(0)$ is the sill of the semi-variogram model and $\gamma$ is the value generated by the semi-variogram model for the vector joining the points $x_{1}$ and $x_{n}$. The weights $\lambda_{n}$ are then used to predict the value of the requested point $x_{q}$ according to (7).

The key strength of Kriging over other deterministic methods is in that the information about spatial relationships is readily included into the weight calculation. On the other hand, Ordinary Kriging is computationally demanding; particularly, the calculation of sample weights is a time-consuming process.

\section{Thinning Algorithms}

The minimum set of measurement points providing the quality assessment with a given accuracy theoretically depends on the way how these points are chosen. In our study, we thus consider two fundamentally different techniques.

1) Probabilistic Thinning: Probabilistic thinning is a simple approach, which has an important feature that it straightforwardly translates into the choice of the measurement points when planning a measurement campaign. In probabilistic thinning, the choice of the points to be removed is made randomly and independently of each other.

2) Deterministic Thinning: An alternative coverage assessment is provided when the measurement points are spread densely and equally over the entire area. Hence, deterministic thinning ensures that for a given number of retained measurement locations, these reside as far from each other as possible. To determine the structure of the measurement points from a random field, we use the following approach: (i) define $M \times M$ grid that specifies squares on the coverage map, (ii) estimate the number of points in each resulting square and sort them in the descending order, (iii) determine how many points need to be removed, e.g., $10 \%$, (iv) remove the measurement points from the square having the highest number of points, such that the number of points therein equals the number of points in the cluster having the secondhighest number of points, (v) keep removing points from the two clusters having the highest numbers of points until when the numbers of points in those equals the number of points in the cluster having the third-highest number of points, and (vi) continue until the required number of points has been removed.

For all the considered interpolation techniques, we employ a similar procedure to identify the set of measurement points that characterize the coverage quality with a certain deviation. Particularly, at each step, we remove $10 \%$ of points and then estimate the coverage-related metrics. Note that the latter approach is deterministic only on the $M \times M$ grid level. The points in each cluster are removed randomly; therefore, the nature of removing points from the individual clusters is probabilistic.

By contrast, the former method is inherently random. It is worth mentioning that the thinning is also connected with the addition of an artificial point to each corner of the interpolated area. Without this step, an extrapolation procedure have to be applied, which we aim to avoid. To this end, the value is set to the minimum sensitivity of each LPWAN technology (signal level of around $-140 \mathrm{dBm}$ ). However, to reduce the impact of the artificial points on the interpolation procedure, we limit their use as much as possible. These points are currently inserted into the input dataset only when the area bounded by the measurement points cannot be fully interpolated (i.e., interpolation returns undefined values). This approach has proven to be reliable when the interpolation is influenced only in the extreme cases of removed points (more than 97\%).

\section{NUMERICAL RESUltS}

In this section, we provide the numerical results and evaluate the considered coverage assessment strategies. Recall that our goal is to assess the accuracy of the proposed algorithm for different densities of measurement points in the environment where the BS locations remain unknown, thus reflecting the precision vs. time consumption trade-off. To this aim, we start by constructing the reference coverage and the signal quality assessment models described in subsection IV-B We then proceed with selecting the appropriate interpolation tools out of those introduced in subsection IV-C

Further, we compare the performance of thinning algorithms specified in subsection IV-D. The presented results should be treated as a comparison of the two models rather than a comparison of the reference model with the results of the proposed methodology. Then, we report on the coverage assessment accuracy with respect to the MAE metric defined in subsection IV-A. We continue by summarizing our insights in the form of a lower bound on the number of measurement points required to deliver accurate coverage assessment. Finally, the section concludes with an accuracy assessment of the proposed models with the measured results, i.e., the correlation between the reference model ("ground truth" data), the predicted values, and the measured data.

\section{A. Comparison of Interpolation Methods}

Before assessing radio coverage and signal quality with a limited number of measurement points as well as comparing the performance of thinning algorithms, we analyze the considered interpolation methods with the goal of selecting 


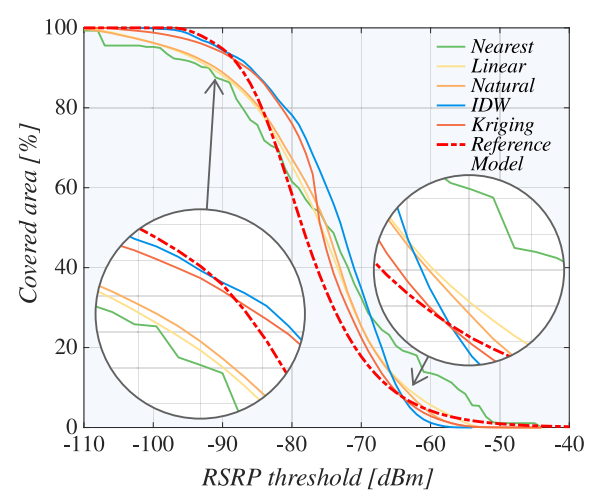

(a) NB-IoT

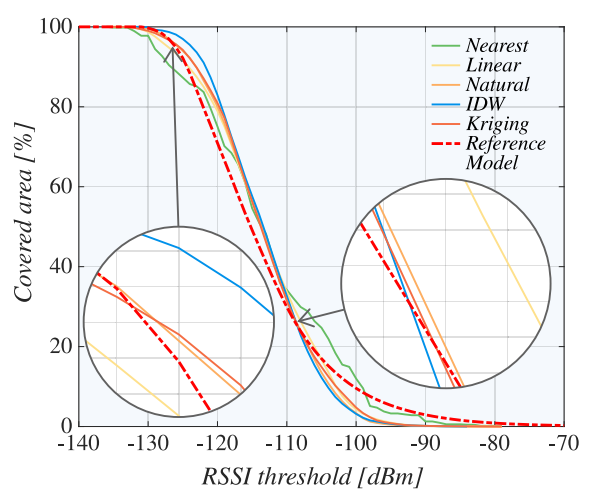

(b) Sigfox

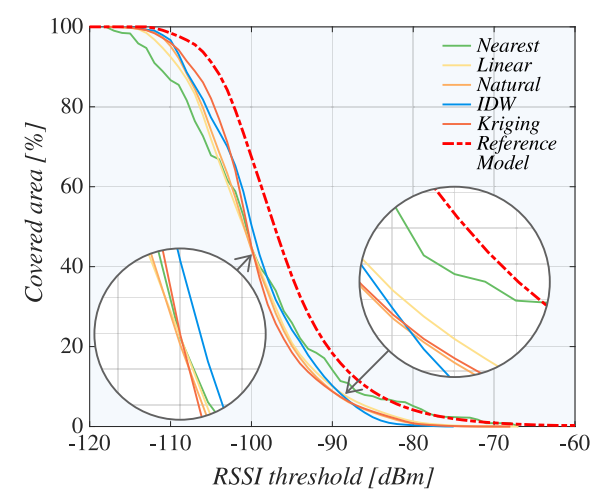

(c) LoRaWAN

Fig. 6: Comparing interpolation methods with reference model.

the algorithms offering the best performance. To this end, we apply the candidate interpolation methods for the scattered input data set to estimate the signal values on a regularly spaced grid. The performance of the interpolation methods is directly compared against the reference model by using the metrics defined in subsection IV-A i.e., inverse CDF and integral MAE parameter (1).

Concerning the CDF values, Fig. 6 offers a visual comparison between the considered interpolation methods and the reference models for all three LPWAN technologies, i.e., NBIoT, Sigfox, and LoRaWAN, by relying on the cumulative percentage of the area featuring the RSRP/RSSI level of at least $x \mathrm{dBm}$ as the parameter of interest. As one may observe, none of the considered interpolation tools provide a perfect fit. However, some methods are significantly better than the other. Notably, the Nearest-neighbor algorithm demonstrates the largest deviation from the reference model out of all the selected interpolation techniques; it is characterized by a sharp decline in the regions of small and high values of RSRP/RSSI.

On the contrary, in the case of NB-IoT, the IDW algorithm offers the worst approximation in the area around the median of the data. It has to also be noted that for NB-IoT, the individual interpolation methods differ from each other most significantly. Two primary facts cause this phenomenon. First, the BS density in the NB-IoT deployment is considerably higher than that for the Sigfox and LoRaWAN layouts, thus influencing the reference model as well as the CDF curve's steepness. Second, the majority of the measurement points oscillate on a short-range scale around the RSRP value of $-75 \mathrm{dBm}$. Hence, the remaining values need to be interpolated from a lower number of samples. For Sigfox and LoRaWAN, the rest of the algorithms display similar outputs.

To numerically quantify the performance of the considered interpolation algorithms, Fig. 7 delivers a comparison with the reference models by using the integral coverage metric defined in (1). Recall that this parameter can be interpreted as a deviation of the coverage metric (RSRP/RSSI) averaged over the set of selected interpolated points. As one may observe, the above conclusions are confirmed numerically. Particularly, the Nearest-neighbor algorithm is characterized by the worst performance out of all the considered interpolation methods.

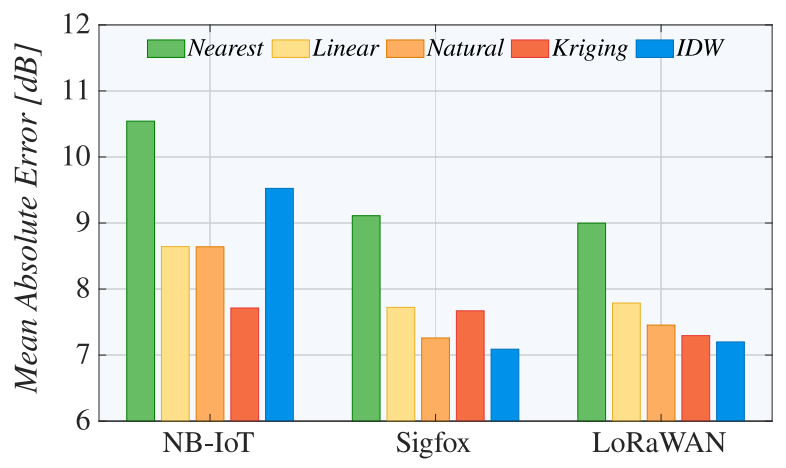

Fig. 7: Mean deviation for interpolation methods.

Even though the IDW option is the second-worst for NBIoT, its results exhibit the lowest deviation in the cases of Sigfox and LoRaWAN technologies. Contrarily, the Kriging algorithm performs as the second-worst for Sigfox and LoRaWAN, whereas in the case of NB-IoT, it is the most reliable option. The remaining Linear and Natural interpolation methods provide satisfactory results for all three considered LPWAN technologies, with Natural interpolation providing a slightly smaller deviation. Surprisingly, all the subject algorithms display comparable results even though their approaches to the interpolated values derivation differ significantly.

Another important observation is that the quality of the resultant approximation heavily depends on the BS deployment density. Analyzing the data presented in Fig. 7 we note that the highest MAE deviation is observed for the NBIoT technology. At the same time, the output for Sigfox and LoRaWAN is noticeably better - this behavior confirms the visual observation gained from Fig. 6 In the considered case, NB-IoT is associated with the densest deployment, while Sigfox relies upon the smallest number of BSs located within the area of interest. We finally note that the averaged deviation as per (1) for the reference model is around $8.5 \mathrm{~dB}$ for NBIoT and approximately $7.4 \mathrm{~dB}$ for Sigfox and LoRaWAN. Hence, one may conclude that the quality of the approximation degrades as the BS deployment density grows. 


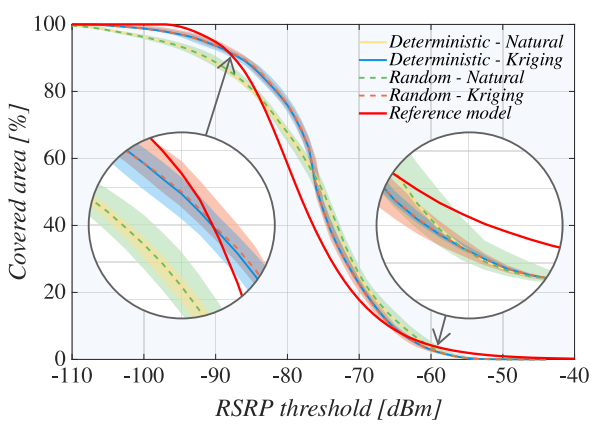

(a) $10 \%$ of removed points

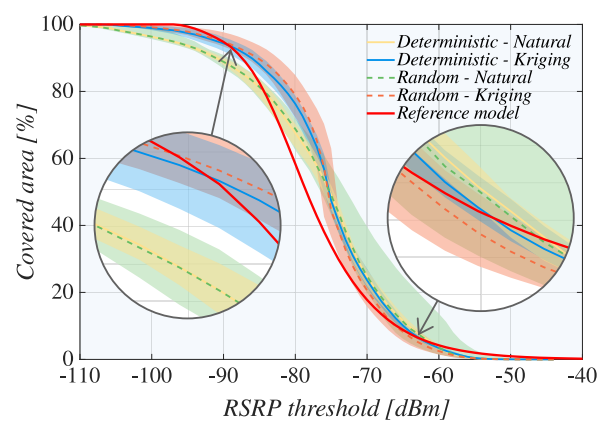

(b) $30 \%$ of removed points

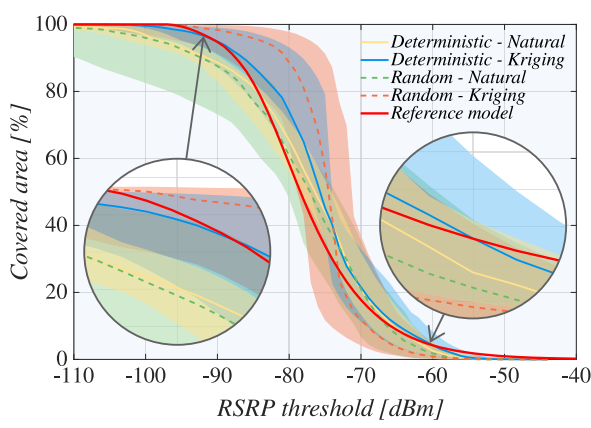

(c) $70 \%$ of removed points

Fig. 8: Performance comparison of interpolation methods and thinning algorithms for NB-IoT.

\section{B. Comparison of Models under Thinning}

In our approach, we target dynamic environments with locations having diverse propagation conditions, where even the use of a precisely fine-tuned model may deliver inaccurate output data. Therefore, the presented results should be considered as a comparison of the two models rather than a performance comparison of the so-called "ground truth" (reference model) with the results of our proposed methodology. To do so, we proceed with contrasting the interpolation algorithms for the thinned data set with a certain fraction of points removed according to the algorithms outlined in subsection IV-D We aim to assess whether the performance of selected interpolation algorithms degrades, and if so, how rapidly this occurs under different percentages of excluded measurement points as the interpolation mechanisms should reflect the changes more precisely.

To this aim, Fig. 8 illustrates the coverage proportion with a given RSRP threshold as a function of the thinning algorithm in use, the type of the employed interpolation, and the number of the removed measurement points. Based on visual analysis, we focus on the NB-IoT technology, as it indicates the highest divergence among all the interpolation methods and reference models. For the sake of clarity, we also select only the two best-performing interpolation methods, i.e., Naturalneighbor and Kriging algorithms. Each subfigure contains the outcomes of 30 subsequent runs combining different thinning and interpolation algorithms. The resulting lines represent the median value based on all the repetitions while the translucent areas denote the $5^{\text {th }}$ and $95^{\text {th }}$ percentiles from left and right, respectively.

One of the essential observations based on the presented data is that the difference in the median values between the thinning algorithms is almost negligible for low numbers of the removed points, i.e., $10 \%$ (30 out of 300) and 30\% (90 out of 300). However, probabilistic thinning is characterized by increased variance, thus resulting in a wider translucent area around the median curve. The difference is even more notable for higher numbers of the removed points, e.g., $70 \%$ (210 out of 300). Even though both thinning algorithms display significantly increased variance, the fluctuation for the deterministic algorithm remains two times smaller.
Surprisingly, the median value disparity between the thinning algorithms is lower for Natural-neighbor interpolation, whereas Kriging method shows significant differences, especially for the RSRP values between -90 and $-75 \mathrm{dBm}$. The Kriging variogram range most probably causes this fact, as it limits the sampling point impact on the interpolated value. Hence, with a more considerable distance between the sampling points, the Kriging option tends to produce bounded areas around the sampling points and settle on the average values in the rest of the plane. In practice, this implies that having a regular structure (such as a grid) for arranging an accurate assessment of the network coverage allows us to significantly decrease the resultant variance, especially for low numbers of the sampling points. Note that similar trends were observed for Sigfox and LoRaWAN deployments.

Further, we report on the behavior of the MAE metric defined in (1), which captures the deviation from the reference models under probabilistic and deterministic thinning of the measurement points for all the considered LPWAN technologies and interpolation methods as illustrated in Fig. 9 and Fig. 10, respectively. As in the previous cases, the results summarize the output of 30 procedure runs, with the barplot head representing the median value and the error bars denoting the $5^{\text {th }}$ and $95^{\text {th }}$ percentiles. Based on the presented results, we may conclude that for the NB-IoT technology, the Kriging algorithm provides the closest approximation of the reference model under both probabilistic and deterministic thinning. Further analysis reveals that the median values for both thinning algorithms are almost identical. In several cases, probabilistic thinning provides even better results, albeit by a narrow margin (in the order of tenths of percents).

If we concentrate on the prediction variance, deterministic thinning provides more coherent results. Logically, this difference increases with higher number of the removed points. On average, probabilistic thinning variance is two times higher regardless of the interpolation algorithm. Hence, by selecting the deterministic algorithm, one can expect more predictable results, which can be slightly biased by the selection of the sampling point locations. This finding is most visible for the IDW interpolation with more than $50 \%$ of the removed points. In this case, probabilistic thinning provides a superior result over the deterministic algorithm. Since IDW predicts 


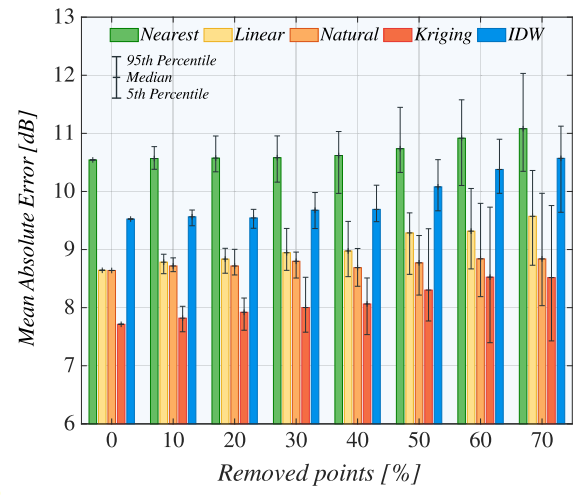

(a) NB-IoT

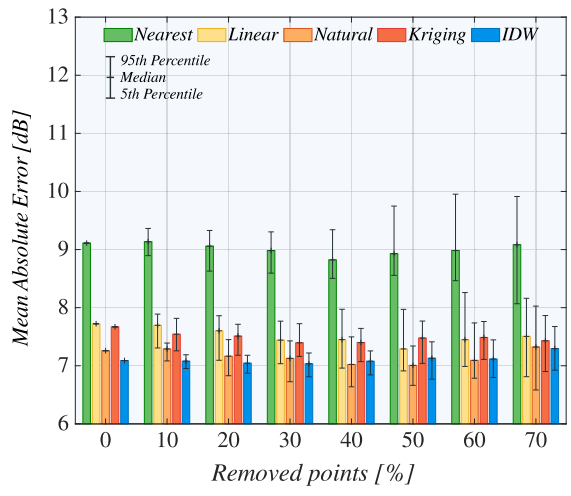

(b) Sigfox

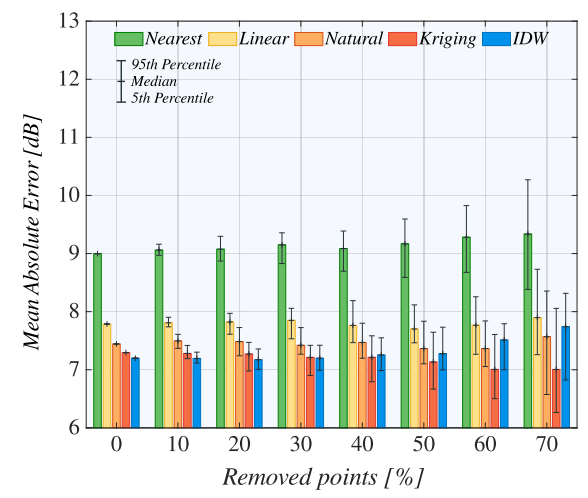

(c) LoRaWAN

Fig. 9: Reference model comparison with interpolated data under deterministic thinning.

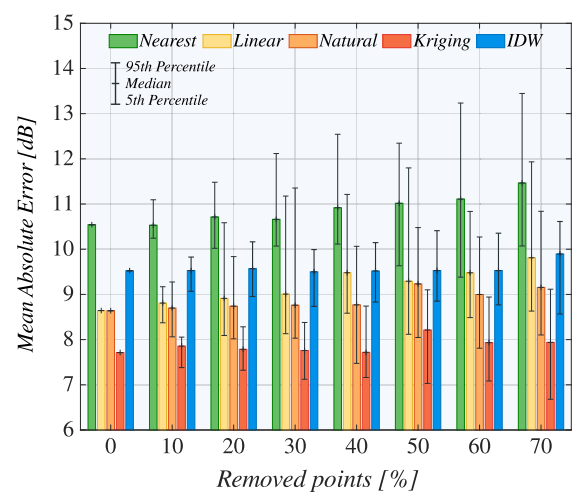

(a) NB-IoT

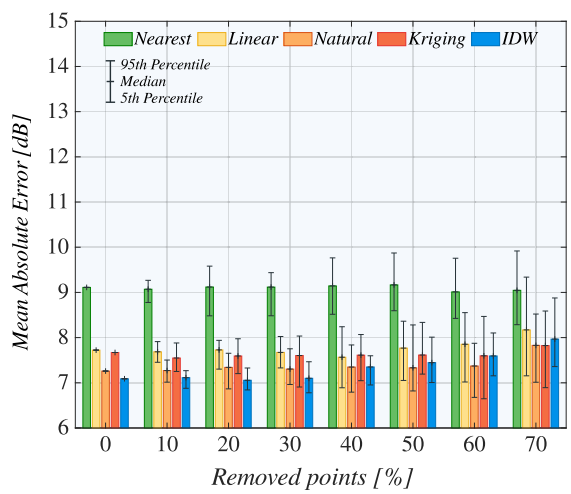

(b) Sigfox

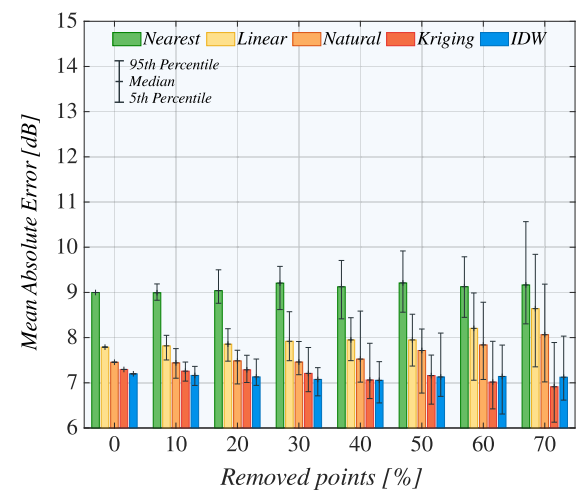

(c) LoRaWAN

Fig. 10: Reference model comparison with interpolated data under probabilistic thinning.

the values from the five closest neighboring points, there is a higher chance of clustering by nearby points when random thinning is applied. On the other hand, deterministic thinning tends to spread the sampling points more evenly; thus, the interpolation must select more distant neighbors with weaker mutual coherence.

Surprisingly, one can observe that IDW interpolation for Sigfox and LoRaWAN technologies under both thinning procedures leads to smaller differences between the considered models. For up to $40 \%$ of the removed points, IDW provides the closest approximation of the reference model for both LPWAN technologies and the considered thinning methods. In the case of Sigfox, for more than $50 \%$ of the removed points, the Natural-neighbor interpolation leads to the best approximation. For LoRaWAN, the Kriging method offers the best approximation of the reference model for $40 \%$ and more points removed. This claim is valid for both deterministic and probabilistic thinning. Another conclusion from the coverage assessment accuracy is that the thinning method selection is not a crucial factor for sparse deployments. With six times sparser deployment of the Sigfox infrastructure as compared to the NB-IoT case, the $5^{\text {th }}$ and $95^{\text {th }}$ percentile variation under deterministic and probabilistic thinning is almost negligible. On the other hand, for the NB-IoT option, the deviation for probabilistic thinning is two times higher.

In summary, one can state these shared characteristics among the results depicted in Fig. 9 and Fig. 10. Clearly, decreasing the number of the measurement points leads to a significantly higher variance in the MAE deviation, thus reducing statistical confidence in the obtained results. The mean deviation is higher for denser deployments represented by the NB-IoT technology. Further, infrastructure density also influences the performance of thinning algorithms. For sparse deployments, the selected thinning method has a marginal impact on the resulting median and variation. The results also suggest that for sparse deployments, such as the ones in Sigfox and LoRaWAN networks, the IDW method provides the best approximation of the reference model for up to $40 \%$ of the removed points (1.2 points per $\mathrm{km}^{2}$ ). On the other hand, for dense cells (e.g., NB-IoT), it is the Kriging algorithm that shows the best approximation capabilities for all the cases of the removed points and thinning algorithms. For the downsides of the methods in question, simple Nearestneighbor interpolation provides the worst approximation of the reference model in all situations. Finally, we note that the MAE deviation metric does not display any sharp increase across the entire range of the removed points from $10 \%(1.8$ points per $\left.\mathrm{km}^{2}\right)$ to $70 \%\left(0.6\right.$ points per $\left.\mathrm{km}^{2}\right)$. 


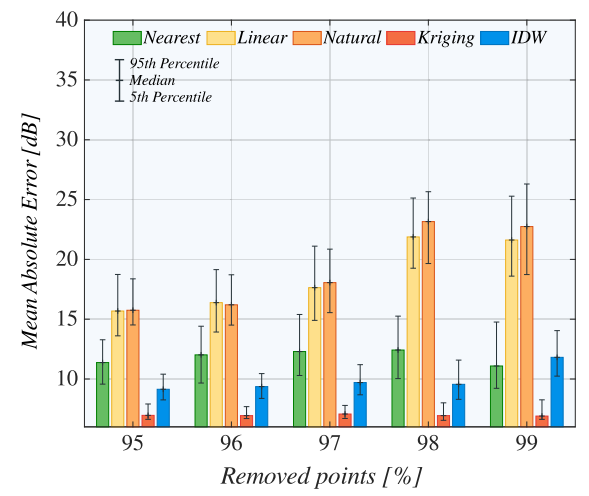

(a) NB-IoT

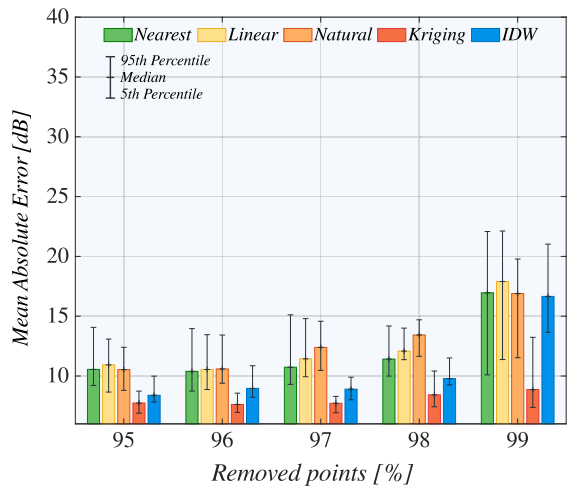

(b) Sigfox

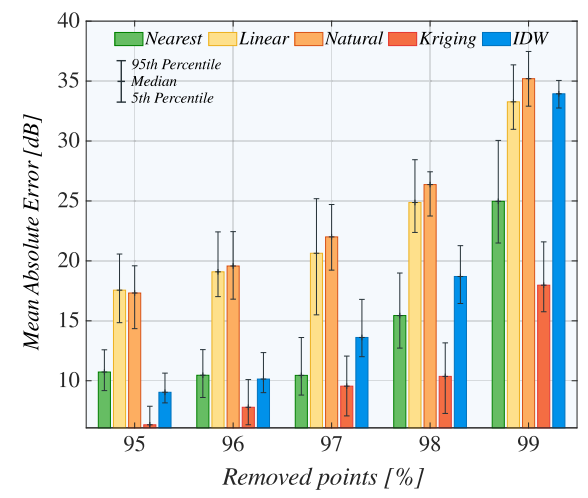

(c) LoRaWAN

Fig. 11: Reference model comparison with interpolated data on lower bound of measurement points with deterministic thinning.

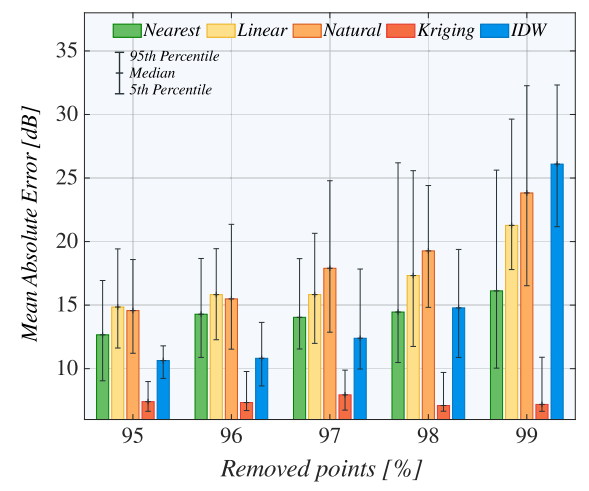

(a) NB-IoT

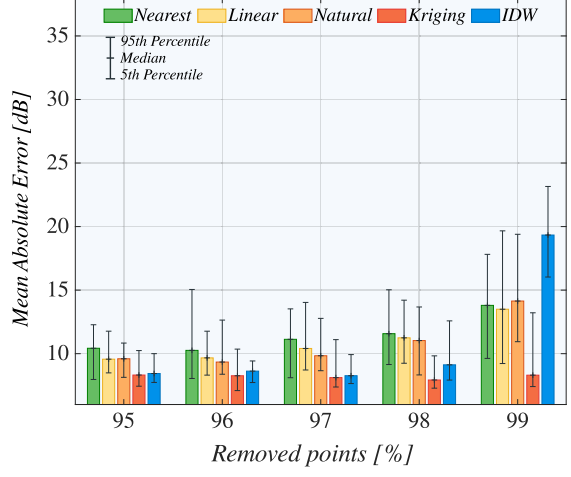

(b) Sigfox

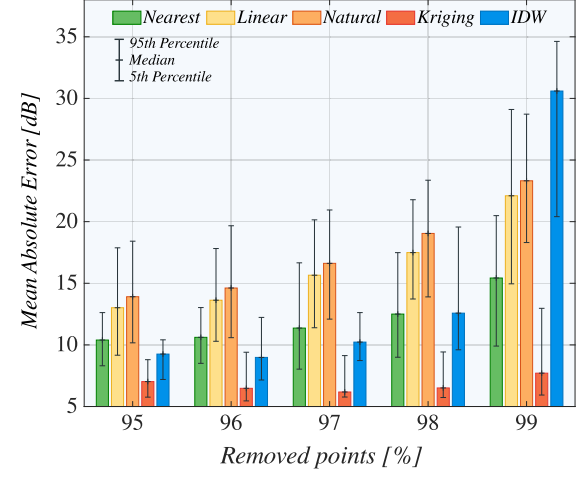

(c) LoRaWAN

Fig. 12: Reference model comparison with interpolated data on lower bound of measurement points with random thinning.

\section{Lower Bound on Number of Measurements}

Since the previous experiments did not identify any particular point where the MAE deviation from the reference model starts to vary significantly, we aimed to relaunch the procedure runs with $95 \%\left(0.1\right.$ points per $\left.\mathrm{km}^{2}\right)$ up to $99 \%$ (0.02 points per $\mathrm{km}^{2}$ ) of the removed points with the goal of producing a lower bound on the number of required measurements. To this end, Fig. 11 and Fig. 12 display the integral metric characterizing the absolute deviation for all the considered interpolation and thinning options.

For the NB-IoT technology, we can see similar characteristics of the thinning algorithms as in the case of Fig. 9 and Fig. 10. Deterministic thinning provides more consistent results than the probabilistic algorithm. Conversely, with the excessive number of removed points, the IDW method under deterministic thinning offers a better approximation of the reference model than the random case. For deterministic thinning, the IDW algorithm further represents the second best approximation, after the Kriging option. However, Linear and Natural-neighbor interpolations indicate more than two times higher mean deviation from the reference model as compared to the initial value ( $0 \%$ of the removed points).

Based on the above, the most promising findings are connected with the Nearest-neighbor and Kriging algorithm. These methods display only a marginal increase in the mean deviation, even for $98 \%$ of the removed points. For the Nearest-neighbor case, this means that the Voronoi diagram with a constant value in each polygon provides a better approximation of the reference model than for the Linear interpolation or the weighted average (Natural-neighbor). In the case of Kriging, the interpolation is solely influenced by the variogram range. If the points are separated by a distance larger than the variogram range, the Kriging method slowly settles to the median value of the samples.

A similar conclusion can be drawn for the LoRaWAN technology. In that case, the Linear and Natural-neighbor interpolations demonstrate the highest mean deviation from the reference model out of all the methods. Also, the Nearestneighbor algorithm provides surprisingly consistent results. As in the previous cases, Sigfox features the most even results out of all the considered technologies. This further confirms that the deployment density significantly impacts the prediction accuracy. Finally, deeper analysis of the results shows that the mean deviation peaks drastically for $98 \%$ or $99 \%$ of the removed points. However, that increase is due to the artificial sample points, which then begin to severely influence the interpolation accuracy.

\section{Accuracy Comparison with Measurement Data}

At the last stage of our numerical evaluation, we compare the accuracy of the values predicted by both the refer- 


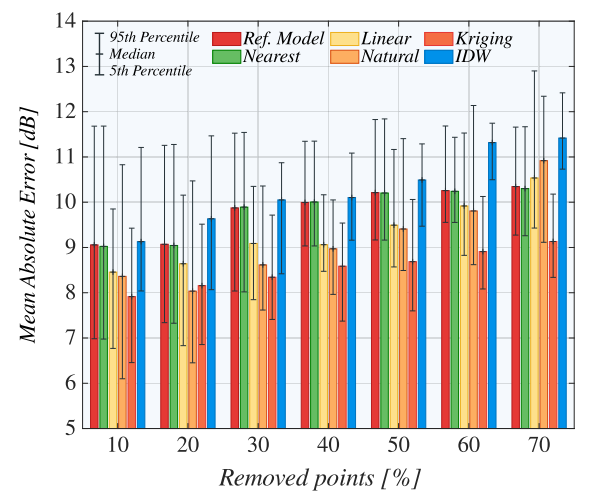

(a) NB-IoT

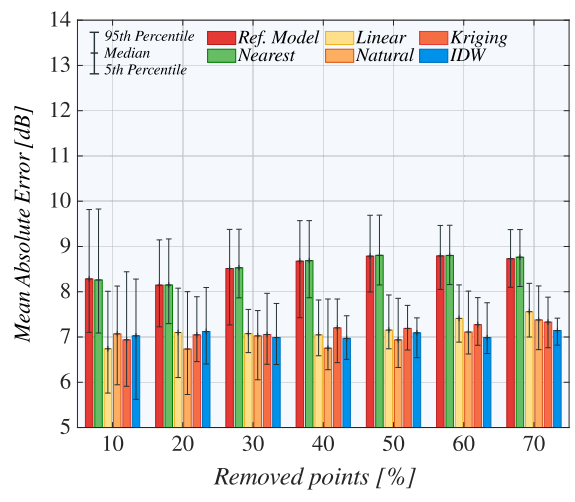

(b) Sigfox

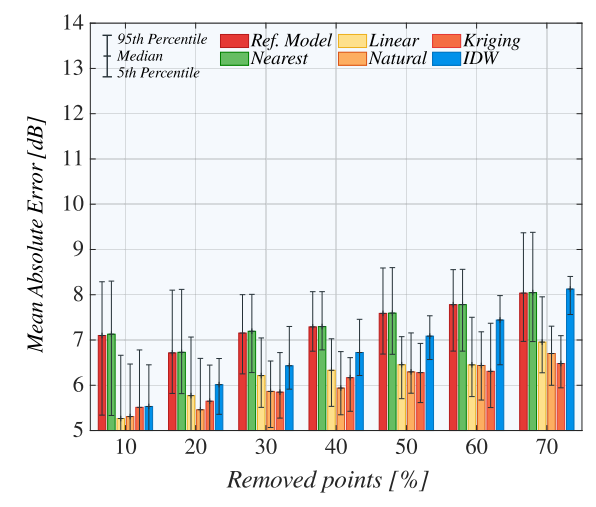

(c) LoRaWAN

Fig. 13: Coverage accuracy assessment for interpolated data with deterministic thinning.

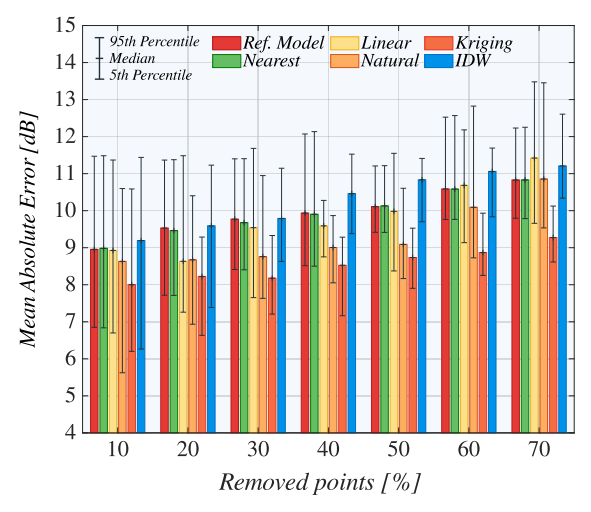

(a) NB-IoT

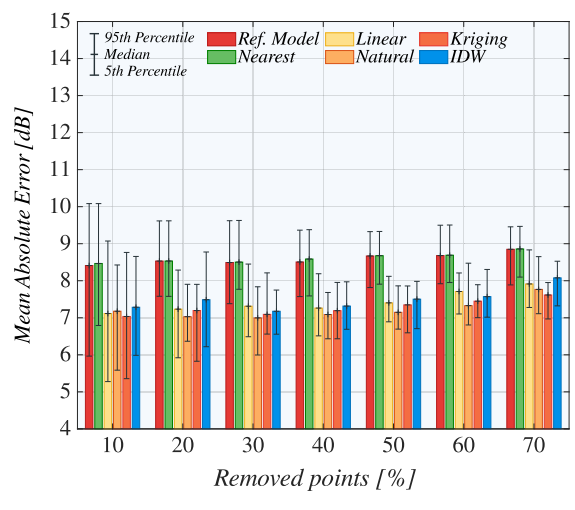

(b) Sigfox

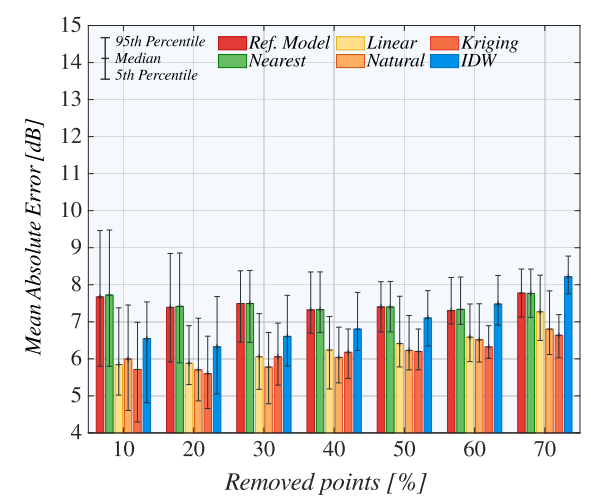

(c) LoRaWAN

Fig. 14: Coverage accuracy assessment for interpolated data with probabilistic thinning.

ence model and the interpolation methods against the actual measurement results. This approach allows us to provide a convenient way of comparing the ground truth data with the predicted values. In summary, with each step of the thinning procedure, the levels of the retracted points are compared to the values predicted by the interpolation algorithms and the reference model. This comparison uses the MAE metric defined in (1). Notably, only a comparison with the removed points is considered since the interpolation methods tend to retain the values of the generating points even for the predicted values. Hence, this would represent an unfair advantage for the interpolation methods if all the measurement points were considered.

A comparison of MAE for both thinning procedures, which is depicted in Fig. 13 and Fig. 14, confirms our earlier assumption that the deployment density influences the prediction accuracy. The NB-IoT technology with the highest BS density out of all the considered options features the increased MAE values by $2-3 \mathrm{~dB}$ on average. These findings are valid for both deterministic and probabilistic thinning. However, the most surprising outcome is related to the performance of the reference model. In most cases, the interpolation methods display notably better results than the fine-tuned propagation model. The two interpolation options that predict values less accurately are the simplest Nearest-neighbor and the IDW.
Further, the only exception is the Sigfox technology, wherein the IDW performs satisfactorily, especially for deterministic thinning.

Conversely, the most versatile interpolation method is the Kriging algorithm, as it provides more accurate results independently of the selected technology, thinning procedure, or the number of retracted measurement points. Our results further indicate that the influence of the thinning methods is negligible. In contrast to the previous comparison with the reference model, the MAE is nearly identical for both deterministic and probabilistic thinning. Even the dispersion of the results is comparable for both approaches. The only exception from this rule is the LoRaWAN technology under deterministic thinning. In that case, MAE linearly increases with the number of retracted points. Notably, the MAE value is nearly constant for the remaining occasions from $10 \%(1.8$ points per $\left.\mathrm{km}^{2}\right)$ to $70 \%\left(0.6\right.$ points per $\left.\mathrm{km}^{2}\right)$ of the removed points.

\section{E. Lower Bound on Number of Measurements Points}

The previous experiments did not reveal any particular point wherein MAE starts to deviate dramatically. Therefore, we here consider the extreme cases of retracting from $95 \%$ $\left(0.1\right.$ points per $\left.\mathrm{km}^{2}\right)$ up to $99 \%\left(0.02\right.$ points per $\left.\mathrm{km}^{2}\right)$ of points. To this end, our main goal is to discover a lower 


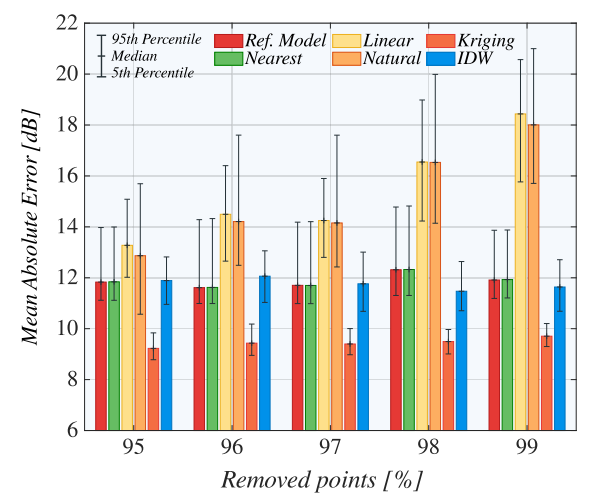

(a) NB-IoT

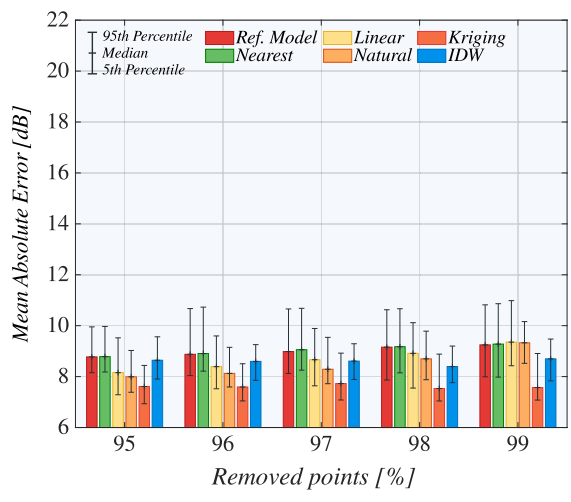

(b) Sigfox

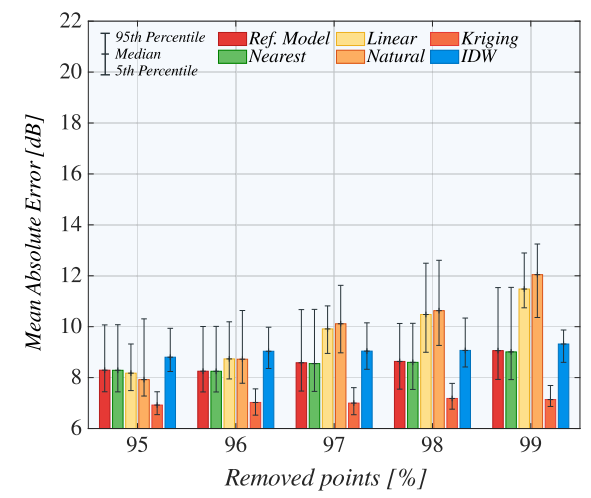

(c) LoRaWAN

Fig. 15: Coverage accuracy assessment on lower bound of measurement points with deterministic thinning.

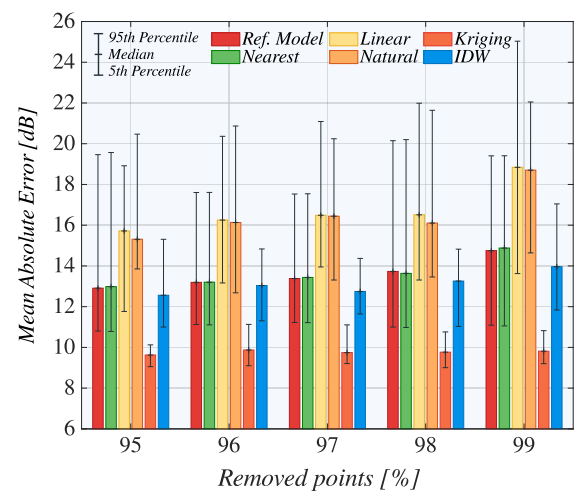

(a) NB-IoT

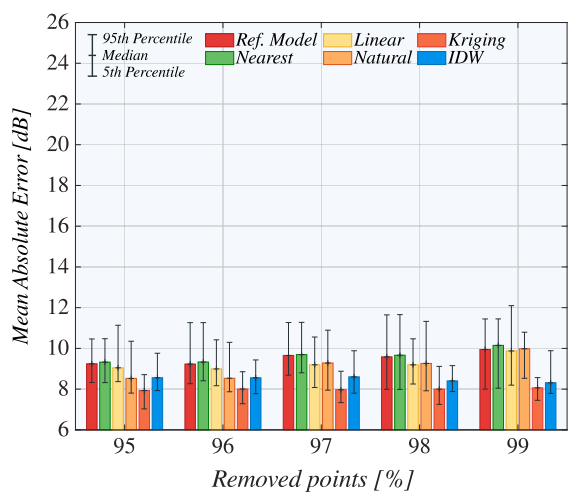

(b) Sigfox

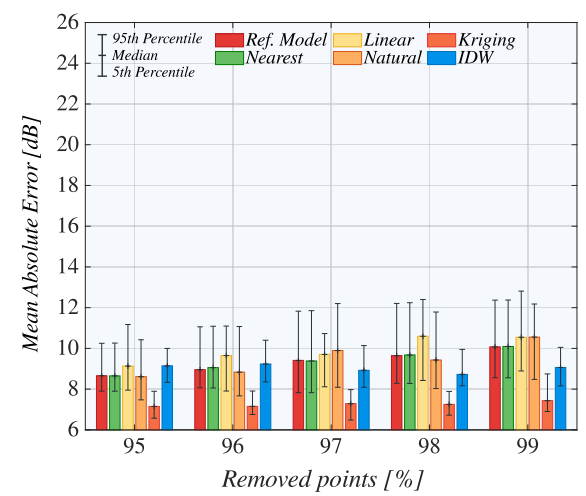

(c) LoRaWAN

Fig. 16: Coverage accuracy assessment on lower bound of measurement points with probabilistic thinning.

bound on the required number of the measurement points, which ensures accurate predictions. The results for the integral metric characterizing the absolute deviation for deterministic and probabilistic thinning methods are depicted in Fig. 15 and Fig. 16, respectively. These results reveal tremendous differences in the prediction accuracy for different LPWA technologies.

The outcomes for high-density NB-IoT deployments show almost two times higher MAE values in comparison to both Sigfox and LoRaWAN cases. Notably, this holds for both deterministic and probabilistic thinning. It is also evident that the Kriging method still holds the first place as the most accurate interpolation option even if compared to the reference model. Surprisingly, for NB-IoT and LoRaWAN, the Linear and Natural-neighbor interpolations display a noticeably increased prediction inaccuracy. For the extreme numbers of the removed points (98\% and 99\%), the presence of artificial "corner" points most probably causes this growth. However, for the lower numbers of the retracted points, the nature of these algorithms is responsible for that effect. In other words, the Linear and Natural-neighbor cases cannot adequately capture the geographical relations between far-away points. On the contrary, the Kriging method, which is specifically designed for this type of correlation, demonstrates its superiority.

\section{CONCLUSIONS}

In this work, we approached the problem of coverage assessment in LPWAN deployments without explicit knowledge of the BS locations. This challenge may be typical for the potential customers who are willing to conduct an access network audit without direct involvement of the network provider. Also, as another example, the operators themselves can be willing to audit the performance of their network to update the layout or configuration. In our study, we primarily concentrated on the three most popular LPWAN technologies, for which the target consideration is particularly essential but the proposed methodology can also be applied to other wireless systems.

We systematically applied various interpolation algorithms to construct the key coverage assessment indicators without the knowledge of BS locations. As an alternative approach, the acquired measurement data can be used to infer the BS locations. This can be done by employing the conventional clusterization mechanisms, e.g., K-means, certain multilateration approaches, or more advanced algorithms based on machine learning. Additionally, we compared these results against the reference model produced by utilizing the experimental results with full BS location knowledge, see Section $\nabla$ for in-depth discussion. 
Using the developed models, we demonstrated that the Kriging interpolation algorithm outperforms its counterparts for NB-IoT deployments with significantly higher BS density. However, for sparser LoRaWAN and Sigfox infrastructures, the performance of simpler interpolation methods, including Linear and Natural-neighbor schemes, is comparable with that of the Kriging algorithm. The IDW technique is significantly more sensitive to the number of measurement points and does not produce accurate approximations in all of the cases. The accuracy of a signal quality assessment heavily depends on the coverage range of the considered radio technology, i.e., the required density of the BS locations. For the considered LPWAN technologies, we can conclude the averaged deviation for the reference model ranges around $8.5 \mathrm{~dB}$ in the case of NB-IoT and oscillates around $7.4 \mathrm{~dB}$ for Sigfox and LoRaWAN.

Notably, the higher is the density, the more significant is the deviation of the interpolation methods from the reference model. Further, we concluded that random selection of the measurement points is as good as deterministic grid measurements. In practice, this implies that the choice of locations wherein measurements are to be made is not critical as long as they essentially cover the entire area of interest. Importantly, there is a limit on the number of measurement points where providing additional volumes of the spatial RSRP/RSSI data does not considerably improve the coverage assessment quality.

Going further, we report on the MAE deviation from the reference models under probabilistic and deterministic thinning, i.e., the behavior of the defined integral metric. For the NB-IoT technology, the Kriging method displays the lowest deviation from the reference model in the case of both thinning procedures. However, if the focus is set on the prediction variance, deterministic thinning comes into play as it provides more coherent results. For Sigfox and LoRaWAN, one may observe dramatically lower MAE values when the IDW option is in use - it is a preferred choice in the scenario with up to $40 \%$ of the removed points. Most importantly, we highlight that the mean deviation metric does not demonstrate any sharp increase across the entire set of the removed points, ranging from $10 \%\left(1.8\right.$ points per $\left.\mathrm{km}^{2}\right)$ to $70 \%\left(0.6\right.$ points per $\left.\mathrm{km}^{2}\right)$.

Hence, we relaunch the procedure runs with $95 \%(0.1$ points per $\mathrm{km}^{2}$ ) up to $99 \%\left(0.02\right.$ points per $\left.\mathrm{km}^{2}\right)$ of the removed points since the previous experiments (see subsection $\mathrm{V}-\mathrm{A}$ and subsection $\mathrm{V}-\mathrm{B}$ ) did not identify any specific evidence where the mean deviation starts to diverge significantly. While analyzing the provided results, one can see that the mean deviation increases for $98 \%$ or $99 \%$ of the removed points. This behavior is connected with the presence of artificial sample points, which at that time influence the interpolation precision.

Finally, we compare the accuracy of the generated models (reference and interpolated options with the measurement data representing the "ground truth"). The output suggests that the accuracy of the results is even less impacted by the selected thinning approach than in the previous cases. It is also evident that most of the employed interpolation methods perform better than the reference model. Particularly, the Kriging algorithm represents the most versatile option among all the interpolation methods, see Section $\mathrm{V}$-D and Section $\mathrm{V}$-E In the case of NB-IoT, the results indicate the highest MAE across all the selected LPWAN technologies, almost two times higher for more than $96 \%$ of the retracted points. These results verify our assumptions related to the fact that the deployment density influences the interpolation performance.

More generally, the telecommunication operators occasionally resort to optimistic coverage maps, which tend to present a better situation than what it actually is in the real-world conditions. Therefore, the end-customers often opt for their own field measurements to understand the current network performance. This approach becomes especially relevant with the development of automated measurement methodologies (e.g., drone-based) and crowd-sourcing concepts (which can also be applied to machines). In these cases, there are essential benefits from the knowledge of the number of points required for an accurate signal and coverage assessment, which is delivered by the present research.

\section{ACKNOWLEDGMENT}

For this research, the infrastructure of the SIX Center was used. This article is based upon the support of international mobility project MeMoV, No. CZ.02.2.69/0.0/0.0/16_027/00083710 funded by the European Union, Ministry of Education, Youth and Sports, Czech Republic, and Brno University of Technology. The work of K. Mikhaylov has been supported by the Academy of Finland 6G Flagship (grant 318927).

\section{REFERENCES}

[1] Sigfox, "Sigfox Connected Objects: Radio Specifications," Sigfox, Ref.: EP-SPECS, Rev.: 1.4, November 2019.

[2] LoRa Alliance ${ }^{\mathrm{TM}}$, "LoRaWAN ${ }^{\mathrm{TM}} 1.1$ Specification," LoRa Alliance ${ }^{\mathrm{TM}}$, Final release, October 2017.

[3] A. Hoglund, X. Lin, O. Liberg, A. Behravan, E. A. Yavuz, M. Van Der Zee, Y. Sui, T. Tirronen, A. Ratilainen, and D. Eriksson, "Overview of 3GPP Release 14 Enhanced NB-IoT," IEEE Network, vol. 31, no. 6, pp. 16-22, November 2017.

[4] U. M. Malik, M. A. Javed, S. Zeadally, and S. ul Islam, "Energy Efficient Fog Computing for 6G Enabled Massive IoT: Recent Trends and Future Opportunities," 2021.

[5] A. Shahraki, M. Abbasi, M. Piran, M. Chen, S. Cui et al., "A Comprehensive Survey on 6G Networks: Applications, Core Services, Enabling Technologies, and Future Challenges," arXiv preprint arXiv:2101.12475, 2021.

[6] A. R. Mishra, Fundamentals of Network Planning and Optimisation 2G/3G/4G: Evolution to 5G. John Wiley \& Sons, 2018.

[7] J. T. Penttinen, The LTE-Advanced Deployment Handbook: The Planning Guidelines for the Fourth Generation Networks. John Wiley \& Sons, 2016.

[8] M. J. Nawrocki, M. Dohler, and A. H. Aghvami, "Modern Approaches To Radio Network Modelling and Planning," Understanding UMTS Radio Network Modelling, Planning and Automated Optimisation: Theory and Practice, p. 3, 2006.

[9] M. Stusek, D. Moltchanov, P. Masek, J. Hosek, S. Andreev, and Y. Koucheryavy, "Learning-Aided Multi-RAT Operation for Battery Lifetime Extension in LPWAN Systems," in 12th International Congress on Ultra Modern Telecommunications and Control Systems and Workshops (ICUMT), 2020, pp. 26-32. 
[10] M. Stusek, D. Moltchanov, P. Masek, S. Andreev, Y. Koucheryavy, and J. Hosek, "Time-Dependent Propagation Analysis and Modeling of LPWAN Technologies," in 2020 IEEE Global Communications Conference, december 2020 , pp. 1-6.

[11] S. Puliti, L. T. Ene, T. Gobakken, and E. Næsset, "Use of Partialcoverage UAV Data in Sampling for Large Scale Forest Inventories," Remote sensing of environment, vol. 194, pp. 115-126, 2017.

[12] B. S. Morse, C. H. Engh, and M. A. Goodrich, "UAV Video Coverage Quality Maps and Prioritized Indexing for Wilderness Search and Rescue," in Proceedings of the 5th ACM/IEEE international conference on Human-robot interaction. IEEE Press, 2010, pp. 227-234.

[13] S. M. Adams and C. J. Friedland, "A Survey of Unmanned Aerial Vehicle (UAV) Usage for Imagery Collection in Disaster Research and Management," in 9th International Workshop on Remote Sensing for Disaster Response, vol. 8, 2011.

[14] M. Brau, Y. Corre, and Y. Lostanlen, "Assessment of 3D Network Coverage Performance From Dense Small-cell LTE," in 2012 IEEE International Conference on Communications (ICC). IEEE, 2012, pp. 6820-6824.

[15] R. Amorim, H. Nguyen, P. Mogensen, I. Z. Kovács, J. Wigard, and T. B. Sørensen, "Radio Channel Modeling for UAV Communication Over Cellular Networks," IEEE Wireless Communications Letters, vol. 6 , no. 4, pp. 514-517, Aug 2017.

[16] M. Stusek, D. Moltchanov, P. Masek, K. Mikhaylov, O. Zeman, M. Roubicek, Y. Koucheryavy, and J. Hosek, "Accuracy Assessment and Cross-Validation of LPWAN Propagation Models in Urban Scenarios," IEEE Access, vol. 8, pp. 154625-154636, 2020.

[17] P. Masek, M. Stusek, K. Zeman, J. Hosek, K. Mikhaylov, S. Andreev, Y. Koucheryavy, O. Zeman, J. Votapek, and M. Roubicek, "Tailoring NB-IoT for Mass Market Applications: A Mobile Operator's Perspective," in 2018 IEEE Globecom Workshops (GC Wkshps). IEEE, 2018, pp. 1-7.

[18] M. Stusek, D. Moltchanov, P. Masek, S. Andreev, Y. Koucheryavy, and J. Hosek, "Time-Dependent Propagation Analysis and Modeling of LPWAN Technologies," in 2020 IEEE Globecom Workshops (GC Wkshps. IEEE, 2020, pp. 1-7.

[19] K. Mikhaylov, M. Stusek, P. Masek, R. Fujdiak, R. Mozny, S. Andreev, and J. Hosek, "On the Performance of Multi-Gateway LoRaWAN Deployments: An Experimental Study," in 2020 IEEE Wireless Communications and Networking Conference (WCNC). IEEE, 2020, pp. $1-6$.

[20] A. Gómez-Andrades, R. Barco, and I. Serrano, "A Method of Assessment of LTE Coverage Holes," EURASIP Journal on Wireless Communications and Networking, vol. 2016, no. 1, p. 236, Oct 2016. [Online]. Available: https://doi.org/10.1186/s13638-016-0733-y

[21] M. Aernouts, R. Berkvens, K. Van Vlaenderen, and M. Weyn, "Sigfox and LoRaWAN Datasets for Fingerprint Localization in Large Urban and Rural Areas," Data, vol. 3, no. 2, p. 13, Apr 2018. [Online]. Available: http://dx.doi.org/10.3390/data3020013

[22] Y. Liu, W. Huangfu, H. Zhang, and K. Long, "Multi-Criteria Coverage Map Construction Based on Adaptive Triangulation-Induced Interpolation for Cellular Networks," IEEE Access, vol. 7, pp. 80767-80777, June 2019.

[23] M. Lauridsen, H. Nguyen, B. Vejlgaard, I. Z. Kovacs, P. Mogensen, and M. Sorensen, "Coverage Comparison of GPRS, NB-IoT, LoRa, and SigFox in a $7800 \mathrm{~km}^{2}$ Area," in 2017 IEEE 85th Vehicular Technology Conference (VTC Spring), June 2017, pp. 1-5.

[24] R. V. Akhpashev and V. G. Drozdova, "Spatial Interpolation of LTE Measurements for Minimization of Drive Tests," in 2018 19th International Conference of Young Specialists on Micro/Nanotechnologies and Electron Devices (EDM), June 2018, pp. 6403-6405.

[25] H. Braham, S. Ben Jemaa, B. Sayrac, G. Fort, and E. Moulines, "Coverage Mapping Using Spatial Interpolation with Field Measurements,' in 2014 IEEE 25th Annual International Symposium on Personal, Indoor, and Mobile Radio Communication (PIMRC), Sep. 2014, pp. 1743-1747.

[26] J. Talvitie, M. Renfors, and E. S. Lohan, "Distance-Based Interpolation and Extrapolation Methods for RSS-Based Localization With Indoor Wireless Signals," IEEE Transactions on Vehicular Technology, vol. 64, no. 4, pp. 1340-1353, April 2015.
[27] M. Chen, Y. Miao, X. Jian, X. Wang, and I. Humar, "CognitiveLPWAN: Towards Intelligent Wireless Services in Hybrid Low Power Wide Area Networks," IEEE Transactions on Green Communications and Networking, vol. 3, no. 2, pp. 409-417, June 2019.

[28] A. Laya, C. Kalalas, F. Vazquez-Gallego, L. Alonso, and J. AlonsoZarate, "Goodbye, ALOHA!" IEEE Access, vol. 4, pp. 2029-2044, April 2016.

[29] P. Masek, M. Stusek, K. Zeman, J. Hosek, K. Mikhaylov, S. Andreev, Y. Koucheryavy, O. Zeman, J. Votapek, and M. Roubicek, "Tailoring NB-IoT for Mass Market Applications: A Mobile Operator's Perspective," in 2018 IEEE Globecom Workshops (GC Wkshps), Dec 2018, pp. $1-7$.

[30] O. Liberg, M. Sundberg, E. Wang, J. Bergman, J. Sachs, and G. Wikström, Cellular Internet of Things: From Massive Deployments to Critical 5G Applications. Academic Press, 2019.

[31] LoRa Alliance ${ }^{\circledR}$, “RP002-1.0.1 ${ }^{\circledR}$ Regional Parameters,” LoRa Alliance ${ }^{\circledR}$, Final, Beaverton, February 2020.

[32] Adeunis, "FTD: Network Tester," Available from: https://www.adeunis. com/, accessed: 2019-01-14.

[33] u-Blox, "SARA-N2 Modules AT Commands Manual," Available from: https://www.u-blox.com/, accessed: 2019-01-14.

[34] RF Solutions Ltd, "GSM Penta Band Antenna," RF Solutions Ltd, ANTPCB8121, November 2011.

[35] M. Stusek, D. Moltchanov, P. Masek, K. Mikhaylov, O. Zeman, M. Roubicek, Y. Koucheryavy, and J. Hosek, "Accuracy Assessment and Cross-Validation of LPWAN Propagation Models in Urban Scenarios," IEEE Access, vol. 8, pp. 154625-154 636, 2020.

[36] 3GPP, "LTE; Evolved Universal Terrestrial Radio Access (E-UTRA); Radio Frequency (RF) System Scenarios (3GPP TR 36.942 Version 15.0.0 Release 15) ," 3GPP, ETSI TR 136942 V15.0.0, July 2018.

[37] MATLAB \& Simulink - MathWorks United Kingdom, "Interpolating Scattered Data - MATLAB \& Simulink," Natick, Massachusetts, USA, 2019. [Online]. Available: https://uk.mathworks.com/help/matlab/math/ interpolating-scattered-data.html

[38] P. Getreuer, "Linear Methods for Image Interpolation," Image Processing On Line, vol. 1, pp. 238-259, 2011.

[39] K. Hormann and N. Sukumar, Generalized Barycentric Coordinates in Computer Graphics and Computational Mechanics. CRC press, 2017.

[40] Y. Diao, Z. Lin, and M. Fu, "A Barycentric Coordinate Based Distributed Localization Algorithm for Sensor Networks," IEEE Transactions on Signal Processing, vol. 62, no. 18, pp. 4760-4771, Sep. 2014.

[41] S. W. Park, L. Linsen, O. Kreylos, J. D. Owens, and B. Hamann, "Discrete Sibson Interpolation," IEEE Transactions on Visualization and Computer Graphics, vol. 12, no. 2, pp. 243-253, March 2006.

[42] R. Sibson, "A Brief Description of Natural Neighbor Interpolation," in Interpolating multivariate data. New York: John Wiley \& Sons, 1981, pp. 21-36.

[43] P. M. Bartier and C. Keller, "Multivariate Interpolation to Incorporate Thematic Surface Data Using Inverse Distance Weighting (IDW)," Computers \& Geosciences, vol. 22, no. 7, pp. 795 - 799, 1996.

[44] D. Shepard, "A Two-dimensional Interpolation Function for Irregularlyspaced Data," in Proceedings of the 1968 23rd ACM National Conference, ser. ACM '68. New York, NY, USA: ACM, 1968, pp. 517-524. [Online]. Available: http://doi.acm.org/10.1145/800186. 810616

[45] Q. Zhang and J. Wu, "Image Super-resolution Using Windowed Ordinary Kriging Interpolation," Optics Communications, vol. 336, pp. 140 - 145, 2015. [Online]. Available: http://www.sciencedirect.com/ science/article/pii/S003040181400889X

[46] A. McBratney and R. Webster, "Choosing Functions for Semivariograms of Soil Properties and Fitting Them to Sampling Estimates," Journal of soil Science, vol. 37, no. 4, pp. 617-639, 1986.

[47] M. R. Inggs and R. T. Lord, "Interpolating Satellite Derived Wind Field Data using Ordinary Kriging, with Application to the Nadir Gap," IEEE Transactions on Geoscience and Remote Sensing, vol. 34, no. 1, pp. 250-256, January 1996. 